\title{
Flux terms and Robin boundary conditions as limit of reactions and potentials concentrating at the boundary
}

\section{José M. Arrieta, Ángela Jiménez-Casas and Aníbal Rodríguez-Bernal}

\begin{abstract}
We analyze the limit of the solutions of an elliptic problem when some reaction and potential terms are concentrated in a neighborhood of a portion $\Gamma$ of the boundary and this neighborhood shrinks to $\Gamma$ as a parameter goes to zero.

We prove that this family of solutions converges in certain Sobolev spaces and also in the sup norm, to the solution of an elliptic problem where the reaction term and the concentrating potential are transformed into a flux condition and a potential on $\Gamma$.
\end{abstract}

\section{Introduction}

Let $\Omega$ be an open bounded smooth set in $\mathbb{R}^{N}$ with a $C^{2}$ boundary $\partial \Omega$. Let $\Gamma \subset \partial \Omega$ be a smooth subset of the boundary, isolated from the rest of the boundary, that is, $\operatorname{dist}(\Gamma, \partial \Omega \backslash \Gamma)>0$.

Define the strip of width $\varepsilon$ and base $\Gamma$ as

$$
\omega_{\varepsilon}=\{x-\sigma \vec{n}(x), x \in \Gamma, \sigma \in[0, \varepsilon)\}
$$

for sufficiently small $\varepsilon$, say $0<\varepsilon<\varepsilon_{0}$, where $\vec{n}(x)$ denotes the outward normal vector. We note that for small $\varepsilon$, the set $\omega_{\varepsilon}$ is a neighborhood of $\Gamma$ in $\bar{\Omega}$, that collapses to the boundary when the parameter $\varepsilon$ goes to zero.

2000 Mathematics Subject Classification: 35J25, 35B20.

Keywords: Elliptic equations, concentrating terms, boundary reaction, boundary potential. 


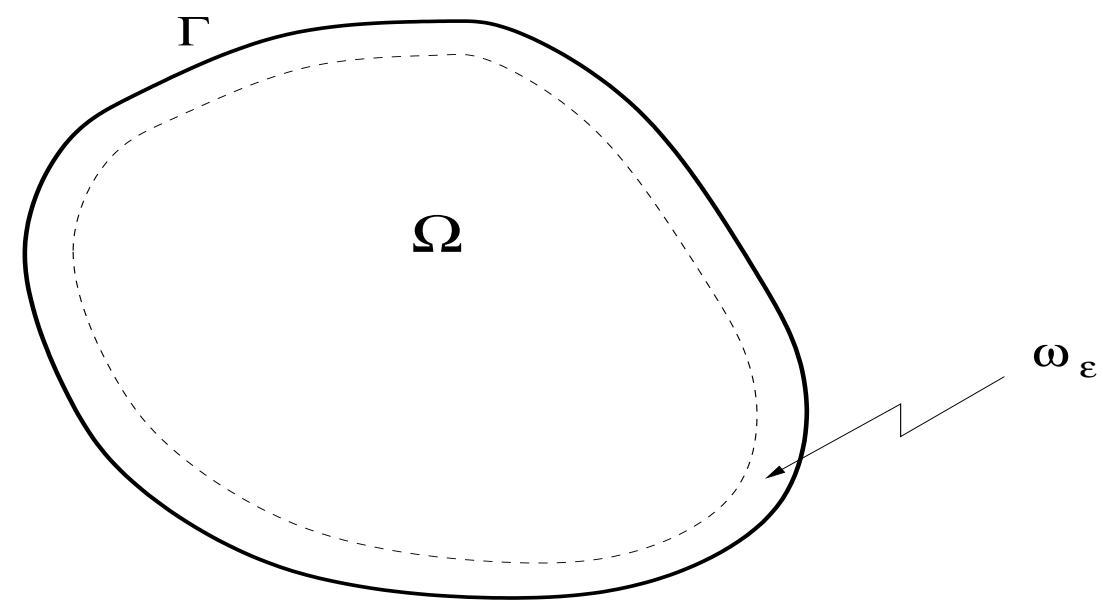

Figure 1: The set $\omega_{\varepsilon}$

We are interested in the behavior, for small $\varepsilon$, of the solutions of an elliptic problem when some reaction terms and some potentials are "concentrated" in $\omega_{\varepsilon}$.

Hence, we consider two family of functions $f_{\varepsilon}$ and $V_{\varepsilon}$ with the property that

$$
\frac{1}{\varepsilon} \int_{\omega_{\varepsilon}}\left|f_{\varepsilon}\right|^{r} \leq C, \quad \frac{1}{\varepsilon} \int_{\omega_{\varepsilon}}\left|V_{\varepsilon}\right|^{\rho} \leq C
$$

for some $1 \leq r, \rho \leq \infty$, where we understand that $r$ or $\rho=\infty$ means that $\left\|f_{\varepsilon}\right\|_{L^{\infty}\left(\omega_{\varepsilon}\right)},\left\|V_{\varepsilon}\right\|_{L^{\infty}\left(\omega_{\varepsilon}\right)}$ are bounded uniformly in $\varepsilon$. Moreover, we assume that there exist functions $f_{0} \in L^{r}(\Gamma)$ and $V_{0} \in L^{\rho}(\Gamma)$ (or bounded Radon measures on $\Gamma, f_{0}, V_{0} \in \mathcal{M}(\Gamma)$ if $r=1=\rho$ ) such that for any smooth function $\varphi$, we have

$$
\lim _{\varepsilon \rightarrow 0} \frac{1}{\varepsilon} \int_{\omega_{\varepsilon}} f_{\varepsilon} \varphi=\int_{\Gamma} f_{0} \varphi, \quad \lim _{\varepsilon \rightarrow 0} \frac{1}{\varepsilon} \int_{\omega_{\varepsilon}} V_{\varepsilon} \varphi=\int_{\Gamma} V_{0} \varphi
$$

For instance, if $f_{\varepsilon} \equiv f \in W^{1, p}(\Omega)$ where $W^{1, p}(\Omega)$ has a well defined trace in $L^{r}(\Gamma)$, then (1.2) and (1.3) hold for $f$ and the function $f_{0}$ is given by the trace of $f$ in $\Gamma$.

Notice also that from condition (1.2) we get that the functions $\frac{1}{\varepsilon} \mathcal{X}_{\omega_{\varepsilon}} f_{\varepsilon}$, $\frac{1}{\varepsilon} \mathcal{X}_{\omega_{\varepsilon}} V_{\varepsilon}$ are uniformly bounded in $L^{1}(\Omega)$, where $\mathcal{X}_{\omega_{\varepsilon}}$ denote the characteristic function of the set $\omega_{\varepsilon}$. We refer to $\frac{1}{\varepsilon} \mathcal{X}_{\omega_{\varepsilon}} f_{\varepsilon}, \frac{1}{\varepsilon} \mathcal{X}_{\omega_{\varepsilon}} V_{\varepsilon}$ as the concentrating functions in $\omega_{\varepsilon}$. 
Hence, we will consider general elliptic problems in divergence form of the type,

$$
\left\{\begin{aligned}
-\operatorname{div}\left(a(x) \nabla u^{\varepsilon}\right)+c(x) u^{\varepsilon}+\lambda u^{\varepsilon}+\frac{1}{\varepsilon} \mathcal{X}_{\omega_{\varepsilon}} V_{\varepsilon}(x) u^{\varepsilon} & =\frac{1}{\varepsilon} \mathcal{X}_{\omega_{\varepsilon}} f_{\varepsilon}+g_{\varepsilon} & & \text { in } \Omega, \\
a(x) \frac{\partial u^{\varepsilon}}{\partial n}+b(x) u^{\varepsilon} & =j_{\varepsilon} & & \text { on } \Gamma, \\
u^{\varepsilon} & =0 & & \text { on } \Gamma_{D},
\end{aligned}\right.
$$

where $\Gamma_{D}=\partial \Omega \backslash \Gamma$ and $\lambda \in \mathbb{R}$. We will show that, for sufficiently large $\lambda$, independent of $\varepsilon$, with $\rho>N-1$, under appropriate values of $r$ and assuming that the terms $g_{\varepsilon}$ and $j_{\varepsilon}$ converge in certain weak sense to $g_{0}$ and $j_{0}$, respectively, then the solutions of (1.4) converge to the unique solution of

$$
\left\{\begin{aligned}
-\operatorname{div}(a(x) \nabla u)+c(x) u+\lambda u & =g_{0} & & \text { in } \Omega \\
a(x) \frac{\partial u}{\partial n}+\left(b(x)+V_{0}(x)\right) u & =f_{0}+j_{0} & & \text { on } \Gamma \\
u & =0 & & \text { on } \Gamma_{D} .
\end{aligned}\right.
$$

We will consider two different cases, according to the smoothness properties of the coefficients of the underlying elliptic operator. If $a, b$ and $c$ are $C^{1}$ functions, we can use the scale of interpolation-extrapolation spaces associated to the elliptic operator, [2]. This scale is given basically by the scale of Bessel potentials $H^{s, p}(\Omega)$, incorporating the boundary conditions accordingly. This scale provides us with a powerful tool to treat perturbations, like the one given by the potentials $\frac{1}{\varepsilon} \mathcal{X}_{\omega_{\varepsilon}} V_{\varepsilon}$ and to consider the concentrated reactions $\frac{1}{\varepsilon} \mathcal{X}_{\omega_{\varepsilon}} f_{\varepsilon}$ as convergent sequences in $H^{-s, p}(\Omega)$ for some appropriate $s, p$. This will allow us to prove that the solutions of (1.4) converge to the unique solution of (1.5) in some Bessel potential spaces $H^{s, p}(\Omega)$ and even in spaces of Hölder functions in $\bar{\Omega}$.

In case the coefficients are not smooth enough, we will rely in the Hilbert space theory and will be able to prove the convergence in $H^{1}(\Omega)$ and, via the De Giorgi-Moser procedure, we will also show the convergence of the solutions in $L^{\infty}(\Omega)$.

Observe that earlier versions of this work, containing weaker results can be found in [5, 6]. Also, [7], contains some related results for some nonlinear eigenvalue problems.

We describe now the contents of the paper. In Section 2 we will prove several technical results on the behavior of concentrating reactions and potentials as $\varepsilon \rightarrow 0$. In Section 3 we deal with the case where the coefficients $a, b$ and $c$ are $C^{1}$. In Section 4 we deal with the case where the coefficients $a, b$ and $c$ are non-smooth. Finally in Section 5 we present several extensions and important applications of the results of this paper. 


\section{Concentrating integrals}

In this section we prove several results that describe how different concentrated integrals converge to surface integrals.

As we mentioned in the introduction, we consider a bounded smooth domain $\Omega \subset \mathbb{R}^{N}$ with smooth boundary $\partial \Omega$ and we also consider $\Gamma \subset \partial \Omega$, a subset of the boundary isolated from the rest of the boundary, that is, $\operatorname{dist}(\Gamma, \partial \Omega \backslash \Gamma)>0$. Then for sufficiently small $\sigma \geq 0$ we can define the "parallel" interior boundary

$$
\Gamma_{\sigma}=\{x-\sigma \vec{n}(x), x \in \Gamma\}
$$

where $\vec{n}(x)$ denotes the outward normal unit at $x \in \Gamma$. Note that $\Gamma_{0}=\Gamma$. Define also the strip of width $\varepsilon$ and base $\Gamma$ as

$$
\omega_{\varepsilon}=\{x-\sigma \vec{n}(x), x \in \Gamma, \sigma \in[0, \varepsilon)\}=\bigcup_{0 \leq \sigma<\varepsilon} \Gamma_{\sigma}
$$

for sufficiently small $\varepsilon$, say $0<\varepsilon<\varepsilon_{0}$.

Note that if we take a continuous function, $v$, in a neighborhood of $\Gamma$, then it is clear that we have

$$
\lim _{\varepsilon \rightarrow 0} \sup _{\omega_{\varepsilon}}|v|=\lim _{\varepsilon \rightarrow 0} \sup _{\Gamma_{\varepsilon}}|v|=\sup _{\Gamma}|v|
$$

In what follows we will be interested in such type of convergence but when certain weighted integral norms are considered.

We will extensively use the scale of Bessel Potential spaces $H^{s, p}(\Omega)$, which are obtained via complex interpolation procedure of the usual Sobolev spaces $W^{k, p}(\Omega)$ with $k=0,1, \ldots$, see for instance $[1,14,2]$. This scale of spaces are suitable to study elliptic and parabolic problems, see [2] for a nice survey on this topic.

Note that the regularity of $\Omega$ and standard trace theory, see [1], imply that for any function $v \in H^{s, p}(\Omega)$, with $s>\frac{1}{p}$, and for any $\sigma \geq 0$ small enough, the trace of $v$ is well defined and it lies in $L^{q}\left(\Gamma_{\sigma}\right)$, provided $s-N / p \geq$ $-(N-1) / q$, that is, with $q \leq \frac{p(N-1)}{N-s p}$. Also in case $s=1=p$ the trace is well defined in $L^{1}\left(\Gamma_{\sigma}\right)$.

The value $\varepsilon_{0}$ above will be chosen small enough so that, for all $0<\varepsilon<\varepsilon_{0}$, the strip $\omega_{\varepsilon}$ can be parameterized in a $C^{2}$ way by $\Gamma \times[0, \varepsilon)$, that is, the map

$$
\begin{aligned}
T_{\varepsilon}: \Gamma \times[0, \varepsilon) & \longrightarrow \omega_{\varepsilon} \\
(x, \sigma) & \longrightarrow x-\sigma \vec{n}(x)
\end{aligned}
$$

is a $C^{2}$ diffeomorphism. 
Notice that if we define $\Omega_{\delta}=\Omega \backslash \bar{\omega}_{\delta}$, for $0<\delta<\varepsilon_{0}$, then we can construct the following $C^{2}$ diffeomorphism $\tau_{\delta}: \bar{\Omega} \longrightarrow \overline{\Omega_{\delta}}$ defined by

$$
\tau_{\delta}(x)= \begin{cases}x & \text { if } \operatorname{dist}(x, \Gamma) \geq \varepsilon_{0} \\ z-\psi_{\delta}(\sigma) \vec{n}(z) & \text { if } x=z-\sigma \vec{n}(z), \sigma \in\left[0, \varepsilon_{0}\right)\end{cases}
$$

where the function $\psi_{\delta}:\left[0, \varepsilon_{0}\right] \rightarrow\left[\delta, \varepsilon_{0}\right]$ is a $C^{2}$ function such that $\psi_{\delta}\left(\varepsilon_{0}\right)=\varepsilon_{0}$, $\psi_{\delta}^{\prime}\left(\varepsilon_{0}\right)=1, \psi_{\delta}^{\prime \prime}\left(\varepsilon_{0}\right)=0, \psi_{\delta}(0)=\delta$, it is strictly increasing, $\left|\psi_{\delta}(\sigma)-\sigma\right|+$ $\left|\psi_{\delta}^{\prime}(\sigma)-1\right|+\left|\psi_{\delta}^{\prime \prime}(\sigma)\right| \rightarrow 0$ uniformly in $\sigma \in\left[0, \varepsilon_{0}\right]$ as $\delta \rightarrow 0$ and the map $\delta \rightarrow \psi_{\delta} \in C^{2}\left(\left[0, \varepsilon_{0}\right]\right)$ is continuous.

Observe that $\tau_{\delta}$ is a $C^{2}$ diffeomorphism between $\Omega$ and $\Omega_{\delta}$ which satisfies $\left\|\tau_{\delta}\right\|_{C^{2}},\left\|\tau_{\delta}^{-1}\right\|_{C^{2}} \leq C$ with $C$ independent of $\delta \in\left(0, \varepsilon_{0}\right)$, the map $\delta \rightarrow \tau_{\delta} \in$ $C^{2}(\bar{\Omega})$ is continuous for $\delta \in\left[0, \varepsilon_{0}\right]$ and $\left\|\tau_{\delta}-I\right\|_{C^{2}(\Omega)} \rightarrow 0$ as $\delta \rightarrow 0$. Note also that $\tau_{\delta}$ is $C^{2}$ diffeomorphism between $\Gamma$ and $\Gamma_{\delta}$

These diffeomorphisms induce isomorphisms $\tau_{\delta}^{*}: H^{s, p}\left(\Omega_{\delta}\right) \longrightarrow H^{s, p}(\Omega)$ for all $0 \leq s \leq 2$ and $1 \leq p \leq+\infty$, which are defined by $\tau_{\delta}^{*}(u)=u \circ \tau_{\delta}$. The $C^{2}$-bounds obtained above for $\tau_{\delta}$ and $\tau_{\delta}^{-1}$ and the fact that $\left\|\tau_{\delta}-I\right\|_{C^{2}(\Omega)} \rightarrow 0$ as $\delta \rightarrow 0$ imply that the isomorphisms $\tau_{\delta}^{*}$ and $\left(\tau_{\delta}^{*}\right)^{-1}$ are uniformly bounded in $\delta \in\left(0, \varepsilon_{0}\right)$. Moreover, we also have that for $u \in H^{s, p}\left(\Omega_{\delta}\right)$, we get $\| \tau_{\delta}^{*}(u)-$ $u \|_{H^{s, p}\left(\Omega_{\delta}\right)} \rightarrow 0$. They also induce the isomorphisms $\hat{\tau}_{\delta}: L^{q}\left(\Gamma_{\delta}\right) \rightarrow L^{q}(\Gamma)$, for $1 \leq q \leq \infty$, defined by $\hat{\tau}_{\delta}(v)=v \circ \tau_{\delta}$. Similarly, as we have argued for $\tau_{\delta}^{*}$ we will have that $\hat{\tau}_{\delta}$ and $\hat{\tau}_{\delta}^{-1}$ are also uniformly bounded. It is not difficult to prove now that if we denote by $\gamma_{\delta}$ the trace operator from $H^{s, p}(\Omega)$ to $L^{q}\left(\Gamma_{\delta}\right)$ and $\gamma$ the trace operator from $H^{s, p}(\Omega)$ to $L^{q}(\Gamma)$ then

$$
\hat{\tau}_{\delta} \circ \gamma_{\delta} \rightarrow \gamma, \quad \text { as } \delta \rightarrow 0
$$

and this convergence is pointwise from $H^{s, p}(\Omega)$ to $L^{q}(\Gamma)$ if $s>1 / p, s-\frac{N}{p} \geq$ $-\frac{N-1}{q}$ and in the operator norm if $s>1 / p, s-\frac{N}{p}>-\frac{N-1}{q}$. Notice also that we have $\hat{\tau}_{\delta} \circ \gamma_{\delta}=\gamma \circ \tau_{\delta}^{*}$

We refer to [7] for more details in the case of $s=1$ and $p=2$.

We can show the following lemma,

Lemma 2.1 Assume that $v \in H^{s, p}(\Omega)$ with $\frac{1}{p}<s \leq 2$ and $s-\frac{N}{p} \geq-\frac{(N-1)}{q}$, or $v \in H^{1,1}(\Omega)$, i.e, $s=1=p$ and $q=1$ below.

Then for sufficiently small $\varepsilon_{0}$, we have

i) The map

$$
\left[0, \varepsilon_{0}\right] \ni \sigma \mapsto \int_{\Gamma_{\sigma}}|v|^{q}
$$

is continuous. 
ii) There exist a positive constant $C$ independent of $\varepsilon$ and $v$ such that for any $\varepsilon \leq \varepsilon_{0}$, we have

$$
\sup _{\sigma \in[0, \varepsilon)}\|v\|_{L^{q}\left(\Gamma_{\sigma}\right)} \leq C\|v\|_{H^{s, p}(\Omega)} .
$$

iii)

$$
\int_{\omega_{\varepsilon}}|v|^{q}=\int_{0}^{\varepsilon}\left(\int_{\Gamma_{\sigma}}|v|^{q}\right) d \sigma .
$$

with the same equality, without the absolute value, if $q=1$.

In particular

$$
\frac{1}{\varepsilon} \int_{\omega_{\varepsilon}}|v|^{q} \leq C\|v\|_{H^{s, p}(\Omega)}^{q}
$$

and

$$
\lim _{\varepsilon \rightarrow 0} \frac{1}{\varepsilon} \int_{\omega_{\varepsilon}}|v|^{q}=\int_{\Gamma}|v|^{q} .
$$

Proof. Throughout the proof we will use the bounds obtained above for the isomorphisms $\tau_{\sigma}^{*}, \hat{\tau}_{\sigma}$ and their inverses.

i) Notice that, under the change of variables given by the diffeomorphism $\tau_{\delta}$, we have

$$
\int_{\Gamma_{\sigma}}|v(z)|^{q} d S_{\sigma}=\int_{\Gamma}\left|v \circ \tau_{\sigma}(x)\right|^{q} J(x, \sigma) d S_{0}
$$

where $S_{\sigma}$ is the surface measure associated to $\Gamma_{\sigma}, 0 \leq \sigma \leq \varepsilon_{0}$ and $J(x, \sigma)$ is the jacobian of the transformation $\tau_{\sigma}: \Gamma \rightarrow \Gamma_{\sigma}$. Notice that $J(x, \sigma)$ is a function involving the first derivatives of the function $\tau_{\sigma}$ and therefore it is a $C^{1}$ function in $x$. Moreover, the map $\sigma \rightarrow J(\cdot, \sigma) \in C^{1}$ is a continuous map. Also, from the smoothness of $\Gamma$ and the definition of $\Gamma_{\delta}$ we have that there exists constants $0<J_{1} \leq J_{2}$ such that $J_{1} \leq J(x, \sigma) \leq J_{2}$ for all $x \in \Gamma$ and for all $\sigma \in\left[0, \varepsilon_{0}\right]$.

If $v \in H^{s, p}(\Omega)$ then $v \circ \tau_{\sigma} \in H^{s, p}(\Omega)$ and the map $\sigma \rightarrow v \circ \tau_{\sigma} \in H^{s, p}(\Omega)$ is continuous. For $\sigma_{0} \in\left[0, \varepsilon_{0}\right]$, we denote by $L_{\sigma_{0}}^{q}(\Gamma)$, the space $L^{q}(\Gamma)$ with the weighted norm given by $J\left(\cdot, \sigma_{0}\right)$, that is $\|f\|_{L_{\sigma_{0}}^{q}(\Gamma)}^{q}=\int_{\Gamma}|f(x)|^{q} J\left(x, \sigma_{0}\right) d S_{0}$. The norm of $L_{\sigma_{0}}^{q}(\Gamma)$ is equivalent to the standard norm (without weight) of $L^{q}(\Gamma)$. Hence, by standard trace theory we have that the embedding $H^{s, p}(\Omega) \rightarrow L_{\sigma_{0}}^{q}(\Gamma)$ is continuous for any fixed $\sigma_{0} \in\left[0, \varepsilon_{0}\right]$. In particular, for any fixed $\sigma_{0} \in\left[0, \varepsilon_{0}\right]$ we have that the map $\sigma \rightarrow \int_{\Gamma}\left|v \circ \tau_{\sigma}(x)\right|^{q} J\left(x, \sigma_{0}\right) d S_{0}$ is continuous. 
To show the continuity of the map $\sigma \rightarrow \int_{\Gamma}\left|v \circ \tau_{\sigma}(x)\right|^{q} J(x, \sigma) d S_{0}$ at $\sigma_{0}$ we notice that

$$
\begin{aligned}
\left.\left|\int_{\Gamma}\right| v \circ \tau_{\sigma}(x)\right|^{q} J(x, \sigma) d S_{0}-\int_{\Gamma}\left|v \circ \tau_{\sigma_{0}}(x)\right|{ }^{q} J\left(x, \sigma_{0}\right) d S_{0} \mid \\
\leq\left.\left|\int_{\Gamma}\right| v \circ \tau_{\sigma}(x)\right|^{q} J\left(x, \sigma_{0}\right) d S_{0}-\int_{\Gamma}\left|v \circ \tau_{\sigma_{0}}(x)\right|^{q} J\left(x, \sigma_{0}\right) d S_{0} \mid \\
\quad+\int_{\Gamma}\left|v \circ \tau_{\sigma}(x)\right|^{q}\left|J(x, \sigma)-J\left(x, \sigma_{0}\right)\right| d S_{0}
\end{aligned}
$$

and both terms go to 0 , the first one by the continuity of the map $\sigma \rightarrow$ $\int_{\Gamma}\left|v \circ \tau_{\sigma}(x)\right|^{q} J\left(x, \sigma_{0}\right) d S_{0}$ and the second one by the continuity of the map $\sigma \rightarrow J(\cdot, \sigma) \in C^{1}(\Gamma)$.

ii) Observe that, if $v \in H^{s, p}(\Omega)$, we have

$$
\begin{aligned}
\left\|\gamma_{\sigma}(v)\right\|_{L^{q}\left(\Gamma_{\sigma}\right)} & \leq C\left\|\hat{\tau}_{\sigma}\left(\gamma_{\sigma}(v)\right)\right\|_{L^{q}(\Gamma)}=C\left\|\gamma\left(\tau_{\sigma}^{*}(v)\right)\right\|_{L^{q}(\Gamma)} \\
& \leq C\left\|\tau_{\sigma}^{*}(v)\right\|_{H^{s, p}(\Omega)} \leq C\|v\|_{H^{s, p}\left(\Omega_{\sigma}\right)} \leq C\|v\|_{H^{s, p}(\Omega)}
\end{aligned}
$$

where $C$ is a constant independent of $v$ and $\sigma \in\left(0, \varepsilon_{0}\right)$. This shows (2.3).

iii) Statement (2.4) follows from Fubini's theorem.

iv) Statement (2.5) follows from (2.3) and (2.4). Finally, statement (2.6) follows from (2.4) and the continuity of (2.2).

Using this result, we can now analyze how concentrating integrals converge for certain families of functions which vary with $\varepsilon$ and have weak regularity properties. More precisely we have the following.

Lemma 2.2 Assume that a given family $f_{\varepsilon}$ defined on $\omega_{\varepsilon}$ satisfies (1.2), that is, for some $1 \leq r<\infty$ and a constant $C$ independent of $\varepsilon$,

$$
\frac{1}{\varepsilon} \int_{\omega_{\varepsilon}}\left|f_{\varepsilon}\right|^{r} \leq C
$$

or

$$
\sup _{x \in \omega_{\varepsilon}}\left|f_{\varepsilon}(x)\right| \leq C
$$

for the case $r=\infty$.

Then, for every sequence converging to zero (that we still denote $\varepsilon \rightarrow 0$ ) there exist a subsequence (that we still denote the same) and a function $f_{0} \in L^{r}(\Gamma)$ (or a bounded Radon measure on $\Gamma, f_{0} \in \mathcal{M}(\Gamma)$ if $r=1$ ) such that 
i) For any smooth function $\varphi$, defined in $\bar{\Omega}$, we have

$$
\lim _{\varepsilon \rightarrow 0} \frac{1}{\varepsilon} \int_{\omega_{\varepsilon}} f_{\varepsilon} \varphi=\int_{\Gamma} f_{0} \varphi
$$

ii) If $u^{\varepsilon} \rightarrow u^{0}$ weakly in $H^{s, p}(\Omega)$ with $s>1 / p$ and

$$
s-\frac{N}{p}>-\frac{N-1}{r^{\prime}},
$$

or strongly in case of equal sign in (2.8), then

$$
\lim _{\varepsilon \rightarrow 0} \frac{1}{\varepsilon} \int_{\omega_{\varepsilon}} f_{\varepsilon} u^{\varepsilon}=\int_{\Gamma} f_{0} u^{0}
$$

Proof. Define, for $s_{0}>\frac{1}{p_{0}}$ and $s_{0}-\frac{N}{p_{0}} \geq-\frac{N-1}{r^{\prime}}$, the linear forms

$$
L_{\varepsilon}(\varphi)=\frac{1}{\varepsilon} \int_{\omega_{\varepsilon}} f_{\varepsilon} \varphi
$$

on $H^{s_{0}, p_{0}}(\Omega)$. Note that Lemma 2.1 and the assumption on $f_{\varepsilon}$ imply that $L_{\varepsilon}$ are well defined and indeed

$$
\left|L_{\varepsilon}(\varphi)\right| \leq\left(\frac{1}{\varepsilon} \int_{\omega_{\varepsilon}}\left|f_{\varepsilon}\right|^{r}\right)^{1 / r}\left(\frac{1}{\varepsilon} \int_{\omega_{\varepsilon}}|\varphi|^{r^{\prime}}\right)^{1 / r^{\prime}} \leq C\|\varphi\|_{H^{s_{0}, p_{0}}(\Omega)}
$$

(with obvious modifications for the case $r=\infty$ ). Hence $\left\{L_{\varepsilon}\right\}$ is bounded in the dual space of $H^{s_{0}, p_{0}}(\Omega)$.

Hence, by the Banach-Alaouglu-Bourbaki theorem and taking subsequences if necessary, we have that there exists $L_{0} \in\left(H^{s_{0}, p_{0}}(\Omega)\right)^{\prime}:=H^{-s_{0}, p_{0}^{\prime}}(\Omega)$ such that

$$
L_{\varepsilon}(\varphi) \rightarrow L_{0}(\varphi), \quad \text { for all } \varphi \in H^{s_{0}, p_{0}}(\Omega)
$$

as $\varepsilon \rightarrow 0$ and the limit is uniform for $\varphi$ in compact sets of $H^{s_{0}, p_{0}}(\Omega)$.

In particular, from (2.10) and Lemma 2.1, we get

$$
\left|L_{0}(\varphi)\right| \leq C\left(\int_{\Gamma}|\varphi|^{r^{\prime}}\right)^{1 / r^{\prime}}
$$

and since traces of $H^{s_{0}, p_{0}}(\Omega)$ are dense in $L^{r^{\prime}}(\Gamma)$ we get that there exist $f_{0} \in L^{r}(\Gamma)$ such that

$$
L_{0}(\varphi)=\int_{\Gamma} f_{0} \varphi
$$

which proves i). Note that if $r=1$ then we obtain $f_{0} \in \mathcal{M}(\Gamma)=(C(\Gamma))^{\prime}$.

Now if $u^{\varepsilon}$ is as in ii) note that, in the case of weak convergence, there exist $s_{0}$ and $p_{0}$ as above such that $H^{s, p}(\Omega) \hookrightarrow H^{s_{0}, p_{0}}(\Omega)$ with compact embedding, which proves ii). In the case of strong convergence, the conclusion follows from the argument leading to $L_{0}$. 
Remark 2.3 Observe that the Lemma above implies that $F_{\varepsilon}=\frac{1}{\varepsilon} \mathcal{X}_{\omega_{\varepsilon}} f_{\varepsilon}$ is a bounded family in $H^{-s, p}(\Omega)=\left(H^{s, p^{\prime}}(\Omega)\right)^{\prime}$ for $s>\frac{1}{p^{\prime}}$ and $s+\frac{N}{p} \geq 1+\frac{N-1}{r}$.

In the following example we characterize the limiting boundary integral in two different situations.

Example 2.4 Assume that $f \in C(\bar{\Omega})$.

i) Define $f_{\varepsilon}=\mathcal{X}_{\omega_{\varepsilon}} f$. Then Lemma 2.2 applies with $1 \leq r \leq \infty$ and $f_{0}=f_{\mid \Gamma}$.

ii) Define $f_{\varepsilon}=\mathcal{X}_{\theta_{\varepsilon}} f$, where $\theta_{\varepsilon} \subset \omega_{\varepsilon}$ is defined as $\theta_{\varepsilon}=\{x-\sigma \vec{n}(x), x \in \Gamma$, $\left.\varepsilon k_{1}(x)<\sigma<\varepsilon k_{2}(x)\right\}$ for some continuous and nonnegative functions $0 \leq$ $k_{1}(x)<k_{2}(x) \leq 1$ defined on $\Gamma$. Then, Lemma 2.2 applies with $1 \leq r \leq \infty$ and $f_{0}(x)=\left(k_{2}(x)-k_{1}(x)\right) f(x)$.

We can also prove,

Lemma 2.5 Assume we have a family of functions $V_{\varepsilon}, 0 \leq \varepsilon \leq \varepsilon_{0}$, verifying the hypotheses of Lemma 2.2. Moreover, assume that (taking subsequences if necessary) there exists a function $V_{0} \in L^{r}(\Gamma)$ (or a bounded Radon measure on $\Gamma, V_{0} \in \mathcal{M}(\Gamma)$ if $r=1$ ) such that for any smooth function $\varphi$, we have

$$
\lim _{\varepsilon \rightarrow 0} \frac{1}{\varepsilon} \int_{\omega_{\varepsilon}} V_{\varepsilon} \varphi=\int_{\Gamma} V_{0} \varphi
$$

Then, for $s>\frac{1}{p}, \sigma>\frac{1}{q}$ and $s+\sigma-\frac{N}{p}-\frac{N}{q}>-\frac{N-1}{r^{\prime}}$, if we define the operators, $P_{\varepsilon}: H^{s, p}(\Omega) \rightarrow H^{-\sigma, q}(\Omega)$ for $0 \leq \varepsilon \leq \varepsilon_{0}$ by

$$
\begin{gathered}
<P_{\varepsilon}(u), \varphi>=\frac{1}{\varepsilon} \int_{\omega_{\varepsilon}} V_{\varepsilon} u^{\varepsilon} \varphi \\
<P_{0}(u), \varphi>=\int_{\Gamma} V_{0} u \varphi,
\end{gathered}
$$

then $P_{\varepsilon} \rightarrow P_{0}$ in $\mathcal{L}\left(H^{s, p}(\Omega), H^{-\sigma, q}(\Omega)\right)$.

Proof. Note that for every $u \in H^{s_{0}, p_{0}}(\Omega)$ and $\varphi \in H^{\sigma_{0}, q_{0}}(\Omega)$ using Lemma 2.1, we have

$$
\begin{aligned}
\left|\frac{1}{\varepsilon} \int_{\omega_{\varepsilon}} f_{\varepsilon} u \varphi\right| \leq & \left(\frac{1}{\varepsilon} \int_{\omega_{\varepsilon}}\left|f_{\varepsilon}\right|^{r}\right)^{\frac{1}{r}}\left(\frac{1}{\varepsilon} \int_{\omega_{\varepsilon}}|u|^{m}\right)^{\frac{1}{m}}\left(\frac{1}{\varepsilon} \int_{\omega_{\varepsilon}}|\varphi|^{n}\right)^{\frac{1}{n}} \\
& \leq C\|u\|_{H^{s_{0}, p_{0}}(\Omega)}\|\varphi\|_{H^{\sigma_{0}, q_{0}}(\Omega)}
\end{aligned}
$$

where $\frac{1}{r}+\frac{1}{m}+\frac{1}{n}=1$ and $r, m, n$ are such that $s_{0}-\frac{N}{p_{0}} \geq-\frac{N-1}{m}$, with $s_{0}>\frac{1}{p_{0}}$, and $\sigma_{0}-\frac{N}{q_{0}} \geq-\frac{N-1}{n}$, with $\sigma_{0}>\frac{1}{q_{0}}$. 
Then the operator $P_{\varepsilon}$ from $H^{s_{0}, p_{0}}(\Omega)$ into $H^{-\sigma_{0}, q_{0}^{\prime}}(\Omega):=\left(H^{\sigma_{0}, q_{0}}(\Omega)\right)^{\prime}$ is uniformly bounded.

Hence, fixed $u \in H^{s_{0}, p_{0}}(\Omega)$ we have by Lemma 2.2 that

$$
<P_{\varepsilon}(u), \varphi>=\frac{1}{\varepsilon} \int_{\omega_{\varepsilon}} V_{\varepsilon} u \varphi \rightarrow \int_{\Gamma} V_{0} u \varphi=<P_{0}(u), \varphi>
$$

uniformly for $\varphi$ in compact sets of $H^{\sigma_{0}, q_{0}}(\Omega)$. Hence if $q \geq q_{0}$ with $\sigma>\frac{1}{q}$ and $\sigma>\sigma_{0}$ then $H^{\sigma, q}(\Omega) \subset H^{\sigma_{0}, q_{0}}(\Omega)$ with compact embedding, and then, in particular

$$
P_{\varepsilon}(u) \rightarrow P_{0}(u) \quad \text { in } \quad H^{-\sigma, q^{\prime}}(\Omega) .
$$

Again this implies uniform convergence for $u$ in compact sets of $H^{s_{0}, p_{0}}(\Omega)$. Hence if $p \geq p_{0}$ with $s>\frac{1}{p}$ and $s>s_{0}$ then $H^{s, p}(\Omega) \subset H^{s_{0}, p_{0}}(\Omega)$ with compact embedding, and then, in particular, we have

$$
P_{\varepsilon} \rightarrow P_{0} \quad \text { in } \quad \mathcal{L}\left(H^{s, p}(\Omega), H^{-\sigma, q^{\prime}}(\Omega)\right)
$$

which gives the result.

\section{Elliptic problems with smooth coefficients}

In this section we analyze the behavior, as $\varepsilon \rightarrow 0$, of the solutions of the elliptic problem (1.4) with smooth coefficients $a, c \in C^{1}(\bar{\Omega}), b \in C^{1}(\Gamma)$, with suitable nonhomogeneous given terms $g_{\varepsilon}, j_{\varepsilon}$ and concentrating potentials $V_{\varepsilon}$ and concentrating nonhomogeneous terms $f_{\varepsilon}$.

Throughout this section we will assume, as in (1.2) and (1.3), that

$$
\frac{1}{\varepsilon} \int_{\omega_{\varepsilon}}\left|f_{\varepsilon}\right|^{r} \leq C, \quad \frac{1}{\varepsilon} \int_{\omega_{\varepsilon}}\left|V_{\varepsilon}\right|^{\rho} \leq C
$$

and that there exist functions $f_{0} \in L^{r}(\Gamma)$ and $V_{0} \in L^{\rho}(\Gamma)$ (or bounded Radon measures on $\Gamma, f_{0}, V_{0} \in \mathcal{M}(\Gamma)$ if $r=1=\rho$ ) such that for any smooth function $\varphi$, we have

$$
\lim _{\varepsilon \rightarrow 0} \frac{1}{\varepsilon} \int_{\omega_{\varepsilon}} f_{\varepsilon} \varphi=\int_{\Gamma} f_{0} \varphi, \quad \lim _{\varepsilon \rightarrow 0} \frac{1}{\varepsilon} \int_{\omega_{\varepsilon}} V_{\varepsilon} \varphi=\int_{\Gamma} V_{0} \varphi,
$$

where $\omega_{\varepsilon}$ is defined by (1.1), see Lemma 2.2 and Lemma 2.5. We will always assume that $\rho>N-1$ and that

$$
\begin{aligned}
& g_{\varepsilon} \rightarrow g_{0} \text { weakly in } L^{z}(\Omega) \\
& j_{\varepsilon} \rightarrow j_{0} \text { weakly in } L^{t}(\Gamma)
\end{aligned}
$$

for some $z, t \geq 1$. 
Now we consider an adequate setting for the elliptic problems (1.4) and (1.5). For this define the elliptic operator $A_{0}$ by $A_{0}(u)=-\operatorname{div}(a(x) \nabla u)+$ $c(x) u$, regarded as an unbounded operator in $X^{0, p}:=L^{p}(\Omega)$, for $1<p<\infty$, with domain given by $X^{1, p}:=\left\{u \in W^{2, p}(\Omega): u=0\right.$ in $\Gamma_{D}, a(x) \frac{\partial u}{\partial n}+b(x) u=$ 0 in $\Gamma$ \}. Using the interpolation-extrapolation techniques in [2], for which the reader is referred for further details, the operator $A_{0}$ has also an associated scale of interpolated Banach spaces $X^{\alpha, p}, 0<\alpha<1$ with the property that $X^{\alpha, p} \hookrightarrow H^{2 \alpha, p}(\Omega)$ where we denote by $H^{s, p}(\Omega)$ the Bessel Potential spaces. We also have that the scale can be extended to the negative exponents with $X^{-\alpha, p}=\left(X^{-\alpha, p^{\prime}}\right)^{\prime}$, for $0<\alpha<1$. Moreover, we have $H^{-2 \alpha, p}(\Omega)=\left(H^{2 \alpha, p^{\prime}}(\Omega)\right)^{\prime} \hookrightarrow X^{-\alpha, p}$.

Now using the different realization of $A_{0}$ in the extrapolated spaces, see [2], problems (1.4) and (1.5) can be written as a perturbation of a fixed elliptic operator, i.e.

$$
A_{0} u^{\varepsilon}+\lambda u^{\varepsilon}+P_{\varepsilon} u^{\varepsilon}=k^{\varepsilon},
$$

for $0 \leq \varepsilon \leq \varepsilon_{0}$. Note that we identify (1.5) with the case $\varepsilon=0$ and $P_{\varepsilon}$ and $P_{0}$ are defined as in Lemma 2.5, that is, $\left\langle P_{\varepsilon} u, \varphi\right\rangle=\frac{1}{\varepsilon} \int_{\omega_{\varepsilon}} V_{\varepsilon} u \varphi$ and $<P_{0} u, \varphi>=\int_{\Gamma} V_{0} u \varphi$. Also, note that $k^{\varepsilon}$ accounts for nonhomogeneous terms and from Lemmas 2.2 and 2.5 we have $P_{\varepsilon} \rightarrow P_{0}$ and $k^{\varepsilon} \rightarrow k$ in some sense.

In fact this convergence will be used to prove that solutions of (1.4) actually converge to solutions of (1.5).

With regards to the behavior of the operators $A_{0}+\lambda I+P_{\varepsilon}$ we have the following

Theorem 3.1 Assume the conditions above and also that $V_{\varepsilon}$ satisfy (3.1) with $\rho>N-1$. Then, there exists some $\lambda_{0}$ independent of $\varepsilon$, such that for $\lambda>\lambda_{0}$ the elliptic operator $A_{0}+\lambda I+P_{\varepsilon}$ in (1.4) is invertible and for $\sigma \in\left(\frac{1}{p^{\prime}}, 2-\frac{1}{p}\right), 0 \leq \varepsilon \leq \varepsilon_{0}$ we have

$$
\left\|\left(A_{0}+\lambda I+P_{\varepsilon}\right)^{-1}\right\|_{\mathcal{L}\left(H^{-\sigma, p}(\Omega), H^{2-\sigma, p}(\Omega)\right)} \leq C,
$$

where $C$ is independent of $\varepsilon$ and $\lambda$.

Proof. With the notations of Lemma 2.5, taking $q=p^{\prime}$, we have that

$$
\begin{aligned}
& <P_{\varepsilon}(u), \varphi>=\frac{1}{\varepsilon} \int_{\omega_{\varepsilon}} V_{\varepsilon} u \varphi, \quad \text { for all } u \in H^{s, p}(\Omega), \varphi \in H^{\sigma, p^{\prime}}(\Omega) \\
& <P_{0}(u), \varphi>=\int_{\Gamma} V_{0} u \varphi, \quad \text { for all } u \in H^{s, p}(\Omega), \varphi \in H^{\sigma, p^{\prime}}(\Omega)
\end{aligned}
$$


is uniformly bounded, for $s>1 / p, \sigma>1 / p^{\prime}$ and

$$
s+\sigma>1+\frac{N-1}{\rho} .
$$

Moreover

$$
P_{\varepsilon} \rightarrow P_{0} \quad \text { in } \quad \mathcal{L}\left(H^{s, p}(\Omega), H^{-\sigma, p}(\Omega)\right) .
$$

Since $\rho>N-1$ we can take $s+\sigma<2$ and then $A_{0}+\lambda I+P_{\varepsilon}$ is well defined from $H^{2-\sigma, p}(\Omega)$ into $H^{-\sigma, p}(\Omega)$ for any $\sigma \in\left(\frac{1}{p^{\prime}}, 2-\frac{1}{p}\right), 0 \leq \varepsilon \leq \varepsilon_{0}$. In particular for given $g \in H^{-\sigma, p}(\Omega)$ the equation $A_{0} u+\lambda u+P_{\varepsilon} u=g$ can be written as

$$
u=\left(A_{0}+\lambda I\right)^{-1}\left(g-P_{\varepsilon} u\right)=\left(A_{0}+\lambda I\right)^{-1} g-\left(A_{0}+\lambda I\right)^{-1} P_{\varepsilon} u=T_{\lambda}^{\varepsilon}(u) .
$$

If $g \in H^{-\sigma, p}(\Omega),\left(A_{0}+\lambda I\right)^{-1} g \in H^{2-\sigma, p}(\Omega)$ and

$$
\left\|\left(A_{0}+\lambda I\right)^{-1} g\right\|_{H^{2-\sigma, p}(\Omega)} \leq C\|g\|_{H^{-\sigma, p}(\Omega)} .
$$

Moreover, from Lemma $2.5, P_{\varepsilon} \rightarrow P_{0}$ in $\mathcal{L}\left(H^{2-\sigma, p}(\Omega), H^{-\tilde{\sigma}, p}(\Omega)\right)$ for some $\tilde{\sigma}<\sigma$ and close to $\sigma$. Using now the resolvent estimates for $A_{0}$, see [3],

$$
\left\|\left(A_{0}+\lambda\right)^{-1}\right\|_{\mathcal{L}\left(H^{-\tilde{\sigma}, p}(\Omega), H^{2-\sigma, p}(\Omega)\right)} \leq \frac{C}{|\lambda|^{(\sigma-\tilde{\sigma}) / 2}},
$$

and we get that the Lipschitz constant of $T_{\lambda}^{\varepsilon}: H^{2-\sigma, p}(\Omega) \rightarrow H^{2-\sigma, p}(\Omega)$ is bounded by $\frac{C}{|\lambda|^{(\sigma-\tilde{\sigma}) / 2}}$. Therefore it is a contraction, with Lipschitz constant $\theta<1$ uniform for large enough $\lambda$ and $0 \leq \varepsilon \leq \varepsilon_{0}$. This implies that the unique fixed point of $T_{\lambda}^{\varepsilon}$ satisfies

$$
\|u\|_{H^{2-\sigma, p}(\Omega)} \leq \frac{1}{1-\theta}\left\|\left(A_{0}+\lambda\right)^{-1} g\right\|_{H^{2-\sigma, p}(\Omega)} \leq \frac{C}{1-\theta}\|g\|_{H^{-\sigma, p}(\Omega)},
$$

which proves the result.

We can prove now,

Corollary 3.2 Assume we are in the conditions of Theorem 3.1 and also that (3.1), (3.2) and (3.3) holds with $\rho>N-1, z \geq N r /(N-1+r)$ and $t \geq r$. Assume also that $\lambda>\lambda_{0}$ is fixed. Then, $u^{\varepsilon}$, the solution of (1.4), satisfies

$$
u^{\varepsilon} \rightarrow u \quad \text { in } \quad H^{s, r}(\Omega)
$$

for any $s<1+\frac{1}{r}$ where $u$ is the unique solution of the limiting problem (1.5).

In particular, if $r>N-1, z>N / 2$ and $t>N-1$, then

$$
u^{\varepsilon} \rightarrow u \quad \text { in } \quad C^{\beta}(\bar{\Omega}) \text {, }
$$

for some $\beta>0$. 
Proof. First note that from Remark 2.3, $F_{\varepsilon}=\frac{1}{\varepsilon} \mathcal{X}_{\omega_{\varepsilon}} f_{\varepsilon}$ is bounded in $H^{-\sigma, r}(\Omega)$ for $\sigma>\frac{1}{r^{\prime}}$. Also, $f_{0}$ belongs to $H^{-\sigma, r}(\Omega)$ for $\sigma>\frac{1}{r^{\prime}}$. Moreover, for the choice of $z$ and $t, g_{\varepsilon} \in L^{z}(\Omega)$ and $j_{\varepsilon} \in L^{t}(\Gamma)$ are also bounded in $H^{-\sigma, r}(\Omega)$ for $\sigma>\frac{1}{r^{\prime}}$ and $0 \leq \varepsilon \leq \varepsilon_{0}$.

Hence, from Theorem 3.1, the solutions of (1.4) and (1.5) are unique and they are bounded in $H^{2-\sigma, r}(\Omega)$, provided we can take $\sigma<2-\frac{1}{r}$. These two conditions are satisfied since we can always choose $\frac{1}{r^{\prime}}<\sigma<2-\frac{1}{r}=1+\frac{1}{r^{\prime}}$.

Notice that choosing $\sigma>1 / r^{\prime}$ but arbitrarily close to $1 / r^{\prime}$, we have that $2-\sigma<1+1 / r$ but it is arbitrarily close to $1+1 / r$. Hence, by Theorem 3.1 the sequence $u_{\varepsilon}$ is bounded in $H^{s, r}(\Omega)$ for all $s<1+\frac{1}{r}$ and therefore it is a relatively compact sequence in these spaces. Hence, under a subsequence we have that $u_{\varepsilon} \rightarrow u \in H^{s, r}(\Omega)$.

Next, we prove $u$ satisfies the elliptic problem (1.5). In effect, multiplying the equation (1.4) by $\varphi \in C_{\Gamma_{D}}^{\infty}(\bar{\Omega})$, the space of $C^{\infty}$ functions in $\bar{\Omega}$ which are identically zero in a neighborhood of $\Gamma_{D}$, we obtain

$$
\begin{gathered}
\int_{\Omega}\left(a(x) \nabla u^{\varepsilon} \nabla \varphi+(c(x)+\lambda) u^{\varepsilon}\right) \varphi+\int_{\Gamma} b(x) u^{\varepsilon} \varphi+\frac{1}{\varepsilon} \int_{w_{\varepsilon}} V_{\varepsilon}(x) u^{\varepsilon} \varphi \\
=\frac{1}{\varepsilon} \int_{\omega_{\varepsilon}} f_{\varepsilon} \varphi+\int_{\Omega} g_{\varepsilon} \varphi+\int_{\Gamma} j_{\varepsilon}^{\varepsilon} \varphi .
\end{gathered}
$$

Now we assume first $\varphi \in C_{c}^{\infty}(\Omega)$ and taking the limit as $\varepsilon$ goes to zero, using (1.3), we get

$$
\int_{\Omega} a(x) \nabla u \nabla \varphi+\int_{\Omega}(c(x)+\lambda) u \varphi=\int_{\Omega} g_{0} u \varphi .
$$

Therefore the limit function satisfies

$$
-\operatorname{div}(a(x) \nabla u)+(c(x)+\lambda) u=g_{0}, \text { in } \Omega .
$$

Now, we consider $\varphi \in C_{\Gamma_{D}}^{\infty}(\bar{\Omega})$ in (3.4) and using (1.3) and the convergence of the traces above, we get

$\int_{\Omega} a(x) \nabla u \nabla \varphi+\int_{\Omega}(c(x)+\lambda) u \varphi+\int_{\Gamma}\left(b(x)+V_{0}\right) u \varphi=\int_{\Omega} g_{0} u \varphi+\int_{\Gamma}\left(f_{0}+j_{0}\right) \varphi$.

Now, since $\lambda>\lambda_{0}$, from the uniqueness of solutions for the limit problem (1.5) obtained above, we have that all the family $u^{\varepsilon}$ converges to $u$.

If $r>N-1, z>N / 2$ and $t>N-1$, we get that, maybe choosing a smaller $r$ but still $r>N-1$ we have $z \geq N r /(N-1+r)$ and $t \geq r$ and therefore $u_{\varepsilon} \rightarrow u_{0}$ in $H^{s, r}(\Omega)$ for all $s<1+\frac{1}{r}$. Hence, the Hölder convergence is obtained using the embedding $H^{s, r}(\Omega) \hookrightarrow C^{\beta}(\bar{\Omega})$ for $s<1+\frac{1}{r}$ but arbitrarily close to it and $r>N-1$. 
We will be able to include a non homogeneous Dirichlet boundary conditions in $\Gamma_{D}$. More precisely we have,

Corollary 3.3 Assume we are in the conditions of Theorem 3.1. Let $h$ be a function defined in $\Gamma_{D}$ such that $h \in H^{1 / 2}\left(\Gamma_{D}\right) \cap C^{\beta}\left(\Gamma_{D}\right)$. Then if in problems (1.4) and (1.5) we substitute the condition $u=0$ on $\Gamma_{D}$ by $u=h$ on $\Gamma_{D}$, then the same conclusions of Corollary 3.2 hold.

Proof. Let us denote by $H$ the unique solution of the following problem

$$
\left\{\begin{aligned}
-\operatorname{div}(a(x) \nabla H)=0 & \text { in } \Omega \\
a(x) \frac{\partial H}{\partial n}=0 & \text { on } \Gamma \\
H=h & \text { on } \Gamma_{D}
\end{aligned}\right.
$$

which, by standard elliptic theory, it is known to exists and $H \in H^{1}(\Omega) \cap$ $C^{\beta}(\bar{\Omega})$.

Rewriting both equations (1.4) and (1.5) in terms of the new variables $v^{\varepsilon}=u^{\varepsilon}-H$ and $v=u-H$, we obtain,

$$
\left\{\begin{aligned}
-\operatorname{div}\left(a(x) \nabla v^{\varepsilon}\right)+c(x) v^{\varepsilon}+\lambda v^{\varepsilon}+\frac{1}{\varepsilon} \mathcal{X}_{\omega_{\varepsilon}} V_{\varepsilon}(x) v^{\varepsilon} & =\frac{1}{\varepsilon} \mathcal{X}_{\omega_{\varepsilon}} \tilde{f}_{\varepsilon}+\tilde{g}_{\varepsilon} & & \text { in } \Omega \\
a(x) \frac{\partial v^{\varepsilon}}{\partial n}+b(x) v^{\varepsilon} & =\tilde{j}_{\varepsilon} & & \text { on } \Gamma \\
v^{\varepsilon} & =0 & & \text { on } \Gamma_{D}
\end{aligned}\right.
$$

and

$$
\left\{\begin{aligned}
-\operatorname{div}(a(x) \nabla v)+c(x) v+\lambda v & =\tilde{g}_{0} & & \text { in } \Omega \\
a(x) \frac{\partial v}{\partial n}+b(x) v+V_{0}(x) v & =\tilde{f}_{0}+\tilde{j}_{0} & & \text { on } \Gamma \\
v & =0 & & \text { on } \Gamma_{D}
\end{aligned}\right.
$$

where

$$
\tilde{f}_{\varepsilon}=f_{\varepsilon}-V_{\varepsilon}(x) H, \quad \tilde{g}_{\varepsilon}=g_{\varepsilon}-(c(x)+\lambda) H, \tilde{j}_{\varepsilon}=j_{\varepsilon}-b(x) h,
$$

and similarly

$$
\tilde{f}_{0}=f_{0}-V_{0}(x) h, \quad \tilde{g}_{0}=g_{0}-(c(x)+\lambda) H, \quad \tilde{j}_{0}=j_{0}-b(x) h .
$$

Applying Corollary 3.2 to problems (3.7) and (3.8) we obtain that $v^{\varepsilon} \rightarrow v$ in $C^{\beta}(\bar{\Omega})$. This implies that $u^{\varepsilon} \rightarrow u$ in $C^{\beta}(\bar{\Omega})$. 


\section{Elliptic problems with nonsmooth coefficients}

In the arguments of the previous section we have used in an essential way that the coefficients in the elliptic operator are smooth so we can use the associated scale of interpolation-extrapolation spaces which are well characterized in terms of the Bessel potential spaces. In case the coefficients are not that smooth, still some analysis can be carried out in a Hilbert space setting. More precisely, we consider the elliptic problem (1.4) with $0<a_{0} \leq a \in L^{\infty}(\Omega)$ and

$$
c \in L^{p}(\Omega), \quad p>\frac{N}{2}, \quad b \in L^{s}(\Gamma), \quad s>N-1 .
$$

Assume, as before, that

$$
\frac{1}{\varepsilon} \int_{\omega_{\varepsilon}}\left|f_{\varepsilon}\right|^{r} \leq C, \quad \frac{1}{\varepsilon} \int_{\omega_{\varepsilon}}\left|V_{\varepsilon}\right|^{\rho} \leq C
$$

and there exist functions $f_{0} \in L^{r}(\Gamma)$ and $V_{0} \in L^{\rho}(\Gamma)$ (or bounded Radon measures on $\Gamma, f_{0}, V_{0} \in \mathcal{M}(\Gamma)$ if $r=1=\rho$ ) such that for any smooth function $\varphi$, we have

$$
\lim _{\varepsilon \rightarrow 0} \frac{1}{\varepsilon} \int_{\omega_{\varepsilon}} f_{\varepsilon} \varphi=\int_{\Gamma} f_{0} \varphi, \quad \lim _{\varepsilon \rightarrow 0} \frac{1}{\varepsilon} \int_{\omega_{\varepsilon}} V_{\varepsilon} \varphi=\int_{\Gamma} V_{0} \varphi .
$$

Moreover, we will always assume that $\rho>N-1$ and that

$$
\begin{aligned}
& g_{\varepsilon} \rightarrow g_{0} \text { weakly in } L^{z}(\Omega) \\
& j_{\varepsilon} \rightarrow j_{0} \text { weakly in } L^{t}(\Omega)
\end{aligned}
$$

for some $z, t \geq 1$ and consider the formal limit problem (1.5).

We have the following,

Theorem 4.1 Assume the above notations and assumptions. Moreover, assume $r>2(N-1) / N, \rho>N-1, z>2 N /(N+2)$ and $t>2(N-1) / N$. Then there exists some $\lambda_{0}$ such that for $\lambda>\lambda_{0}$ the family of solutions of (1.4), $u^{\varepsilon}$, converges in $H^{1}(\Omega)$, as $\varepsilon \rightarrow 0$, to the unique solution of (1.5). Even more $\lambda_{0}=0$ if $b, c, V_{\varepsilon} \geq 0$.

Proof. We split the proof in several steps.

Step 1 We prove that there exists $\lambda_{0}$ such that for $\lambda>\lambda_{0}$ the bilinear forms in $H^{1}(\Omega)$

$$
a_{\varepsilon}(\phi, \xi)=\int_{\Omega} a(x) \nabla \phi \nabla \xi+\int_{\Omega}(c(x)+\lambda) \phi \xi+\int_{\Gamma} b(x) \phi \xi+\frac{1}{\varepsilon} \int_{\omega_{\varepsilon}} V_{\varepsilon} \phi \xi
$$


and

$$
a_{0}(\phi, \xi)=\int_{\Omega} a(x) \nabla \phi \nabla \xi+\int_{\Omega}(c(x)+\lambda) \phi \xi+\int_{\Gamma}\left(b(x)+V_{0}(x)\right) \phi \xi
$$

are uniformly coercive. In particular, from the choice of $r, z$ and $t$, there exists a unique solution in $H^{1}(\Omega)$ of $(1.4)$ and (1.5).

For this note that for every $\phi \in H^{1}(\Omega)$ we have that, for the negative parts we have the bound

$$
\frac{1}{\varepsilon} \int_{\omega_{\varepsilon}}\left(V_{\varepsilon}\right)_{-}|\phi|^{2} \leq\left(\frac{1}{\varepsilon} \int_{\omega_{\varepsilon}}\left|\left(V_{\varepsilon}\right)_{-}\right|^{\rho}\right)^{\frac{1}{\rho}}\left[\frac{1}{\varepsilon} \int_{\omega_{\varepsilon}}|\phi|^{2 \rho^{\prime}}\right]^{\frac{1}{\rho^{\prime}}} .
$$

Now, since $\rho>N-1$, from Lemma 2.5, with $p=2=q, r=\rho$, we have, for some $s=\sigma<1$ and $s \geq \frac{N-1}{2 \rho}+\frac{1}{2}$

$$
\frac{1}{\varepsilon} \int_{\omega_{\varepsilon}}\left(V_{\varepsilon}\right)_{-}|\phi|^{2} \leq C\|\phi\|_{H^{s}(\Omega)}^{2} \leq C\|\phi\|_{H^{1}(\Omega)}^{2 s}\|\phi\|_{L^{2}(\Omega)}^{2(1-s)} .
$$

Finally using Young's inequality, we get for any $\delta>0$

$$
\frac{1}{\varepsilon} \int_{\omega_{\varepsilon}}\left(V_{\varepsilon}\right)_{-}|\phi|^{2} \leq \delta\|\phi\|_{H^{1}(\Omega)}^{2}+C_{\delta}\|\phi\|_{L^{2}(\Omega)}^{2} .
$$

On the other hand, from Holder's inequality we have

$$
\int_{\Omega} c_{-}(x)|\phi|^{2} \leq \int_{\Omega} c_{-}(x)|\phi|^{2} \leq\left\|c_{-}\right\|_{L^{p}(\Omega)}\|\phi\|_{L^{2 p^{\prime}}(\Omega)}^{2} .
$$

Since $p>\frac{N}{2}$ we have that $H^{1}(\Omega) \subset L^{2 p^{\prime}}(\Omega)$, and there exist $0<s<1$ such that $H^{s}(\Omega) \subset L^{2 p^{\prime}}(\Omega)$, and then we have

$$
\int_{\Omega} c_{-}(x)|\phi|^{2} \leq \delta\|\phi\|_{H^{1}(\Omega)}^{2}+C_{\delta}\|\phi\|_{L^{2}(\Omega)}^{2}
$$

with $\delta<<1$ and $C_{\delta}$ independent of $\varepsilon$.

Finally, since $s>N-1$, we have $H^{1}(\Omega) \subset L^{2 s^{\prime}}(\Gamma)$ and there exits $0<s<1$ such that $H^{s}(\Omega) \subset L^{2 s^{\prime}}(\Gamma)$ and then we have that

$$
\begin{aligned}
\int_{\Gamma} b_{-}(x)|\phi|^{2} & \leq\left\|b_{-}\right\|_{L^{s}(\Gamma)}\left\|\phi^{2}\right\|_{L^{s^{\prime}}(\Gamma)}=\left\|b_{-}\right\|_{L^{s}(\Gamma)}\|\phi\|_{L^{2 s^{\prime}}(\Gamma)}^{2} \\
& \leq \delta\|\phi\|_{H^{1}(\Omega)}^{2}+C_{\delta}\|\phi\|_{L^{2}(\Omega)}^{2} .
\end{aligned}
$$

Hence, we can take $\delta$ small enough and $\lambda$ large enough such that

$$
\begin{aligned}
a_{\varepsilon}(\phi, \phi)= & \int_{\Omega} a(x)|\nabla \phi|^{2}+\int_{\Omega}(c(x)+\lambda)|\phi|^{2}+ \\
& +\int_{\Gamma} b(x)|\phi|^{2}+\frac{1}{\varepsilon} \int_{\omega_{\varepsilon}} V_{\varepsilon}|\phi|^{2} \geq C\|\phi\|_{H^{1}(\Omega)}^{2}
\end{aligned}
$$

with $C=C(\lambda)>0$ independent of $\varepsilon$. 
A similar argument using that $V_{0} \in L^{\rho}(\Gamma)$ and $\rho>N-1$ gives the result for the bilinear form $a_{0}$.

Step 2 For $\lambda>\lambda_{0}$, the family $u^{\varepsilon}$ is uniformly bounded with respect to $\varepsilon$ in $H^{1}(\Omega)$.

In fact from (1.4) we have

$$
a_{\varepsilon}\left(u^{\varepsilon}, u^{\varepsilon}\right)=\frac{1}{\varepsilon} \int_{\omega_{\varepsilon}} f_{\varepsilon} u^{\varepsilon}+\int_{\Omega} g_{\varepsilon} u^{\varepsilon}+\int_{\Gamma} j_{\varepsilon} u^{\varepsilon} .
$$

From Remark 2.3, with $s=1, p=2$, or (2.10), we get that, for sufficiently small $\delta>0$,

$$
\left|\frac{1}{\varepsilon} \int_{\omega_{\varepsilon}} f_{\varepsilon} u^{\varepsilon}\right| \leq c\left\|u^{\varepsilon}\right\|_{H^{1}(\Omega)} \leq \delta\left\|u^{\varepsilon}\right\|_{H^{1}(\Omega)}^{2}+C_{\delta}
$$

since $r \geq \frac{2(N-1)}{N}$. On the other hand, from the assumptions on $z$ and $t$, we have

$$
\left|\int_{\Omega} g_{\varepsilon} u^{\varepsilon}\right| \leq\left\|g_{\varepsilon}\right\|_{L^{z}(\Omega)}\left\|u^{\varepsilon}\right\|_{L^{z^{\prime}(\Omega)}} \leq \delta\left\|u^{\varepsilon}\right\|_{H^{1}(\Omega)}^{2}+C_{\delta}\left\|g_{\varepsilon}\right\|_{L^{z}(\Omega)}^{2}
$$

and

$$
\left|\int_{\Gamma} j_{\varepsilon} u^{\varepsilon}\right| \leq\left\|j_{\varepsilon}\right\|_{L^{t}(\Gamma)}\left\|u^{\varepsilon}\right\|_{L^{t^{\prime}(\Gamma)}} \leq \delta\left\|u^{\varepsilon}\right\|_{H^{1}(\Omega)}^{2}+C_{\delta}\left\|j_{\varepsilon}\right\|_{L^{t}(\Gamma)}^{2} .
$$

From this, using the boundedness of $\left\|g_{\varepsilon}\right\|_{L^{z}(\Omega)}$ and $\left\|j_{\varepsilon}\right\|_{L^{t}(\Gamma)}$ and (4.7) we obtain

$$
\left\|u^{\varepsilon}\right\|_{H^{1}(\Omega)}^{2} \leq C
$$

for some $C$ independent of $\varepsilon$.

From this, there exists a subsequence that we still denote $u^{\varepsilon}$ which converges weakly to $u$ in $H^{1}(\Omega)$. Moreover, from Sobolev's embedding, we can assume the subsequence converges also strongly in $H^{s}(\Omega)$, with $s<1$, and in $L^{p}(\Omega)$ with $p<\frac{2 N}{N-2}$ and almost everywhere. Even more, we can assume the traces converge in $L^{q}(\Gamma)$ for $q<\frac{2(N-1)}{N-2}$ and almost everywhere on $\Gamma$.

Step 3 The limit function satisfies (1.5). From this and the uniqueness of solutions of (1.5), we get that the whole family $u^{\varepsilon}$ converges to $u$.

Note that from (1.5), for any smooth test function $\varphi \in C^{\infty}(\bar{\Omega})$ we obtain

$$
\begin{gathered}
\int_{\Omega} a(x) \nabla u^{\varepsilon} \nabla \varphi+\int_{\Omega}(c(x)+\lambda) u^{\varepsilon} \varphi+\int_{\Gamma} b(x) u^{\varepsilon} \varphi+\frac{1}{\varepsilon} \int_{\omega_{\varepsilon}} V_{\varepsilon}(x) u^{\varepsilon} \varphi \\
=\frac{1}{\varepsilon} \int_{\omega_{\varepsilon}} f_{\varepsilon} \varphi+\int_{\Omega} g_{\varepsilon} \varphi+\int_{\Gamma} j_{\varepsilon} \varphi .
\end{gathered}
$$


Now note that since $\rho>N-1$, from Lemma 2.5, with $p=2=q, r=\rho$ and for some $s=\sigma<1$ we can pass to the limit in the first term of the right hand side. This and the convergence of $u^{\varepsilon}$ in Step 2, allows us to pass to the limit above, to get

$\int_{\Omega} a(x) \nabla u \nabla \varphi+\int_{\Omega}(c(x)+\lambda) u \varphi+\int_{\Gamma}\left(b(x)+V_{0}(x)\right) u \varphi=\int_{\Omega} g_{0} \varphi+\int_{\Gamma}\left(j_{0}+f_{0}\right) \varphi$.

Hence, $u$ is the weak solution of the limit problem (1.5).

Step 4 Now we prove that $u^{\varepsilon}$ converges to $u$ strongly in $H^{1}(\Omega)$. Note that for this it is enough to prove convergence of the norms.

For this, note that in (4.8), using the convergence from Step 2 and Lemma 2.2 with $s=1, p=2$ and $r>\frac{2(N-1)}{N}$, we have

$$
\lim _{\varepsilon \rightarrow 0}\left(\frac{1}{\varepsilon} \int_{\omega_{\varepsilon}} f_{\varepsilon} u^{\varepsilon}+\int_{\Omega} g_{\varepsilon} u^{\varepsilon}+\int_{\Gamma} j_{\varepsilon} u^{\varepsilon}\right)=\int_{\Gamma} f_{0} u+\int_{\Omega} g_{0} u+\int_{\Gamma} j_{0} u
$$

and also

$$
\begin{aligned}
& \lim _{\varepsilon \rightarrow 0} \int_{\Omega}(c(x)+\lambda)\left|u^{\varepsilon}\right|^{2}=\int_{\Omega}(c(x)+\lambda)|u|^{2}, \\
& \lim _{\varepsilon \rightarrow 0} \int_{\Gamma} b(x)\left|u^{\varepsilon}\right|^{2}=\int_{\Gamma} b(x)|u|^{2} .
\end{aligned}
$$

On the other hand, using again $\rho>N-1$, from Lemma 2.5, with $p=2=q$, $r=\rho$ and for some $s=\sigma<1$, we get

$$
\lim _{\varepsilon \rightarrow 0} \frac{1}{\varepsilon} \int_{\omega_{\varepsilon}} V_{\varepsilon}(x)\left|u^{\varepsilon}\right|^{2}=\int_{\Gamma} V_{0}(x)|u|^{2} .
$$

With this, passing to the limit in (4.8), we get

$$
\begin{aligned}
\int_{\Omega} a(x)|\nabla u|^{2} & \leq \liminf _{\varepsilon} \int_{\Omega} a(x)\left|\nabla u^{\varepsilon}\right|^{2} \leq \limsup _{\varepsilon} \int_{\Omega} a(x)\left|\nabla u^{\varepsilon}\right|^{2} \\
& \leq-\int_{\Omega}(c(x)+\lambda)|u|^{2}-\int_{\Gamma} V_{0}(x)|u|^{2}+\int_{\Gamma}\left(j+f_{0}\right) u+\int_{\Omega} g_{0} u .
\end{aligned}
$$

Finally from the variational formulation of the limit problem we have

$$
\int_{\Omega} a(x)|\nabla u|^{2}=-\int_{\Omega}(c(x)+\lambda)|u|^{2}-\int_{\Gamma} V_{0}(x)|u|^{2}+\int_{\Gamma}\left(j+f_{0}\right) u+\int_{\Omega} g_{0} u
$$

and we conclude. 
In particular, from this we can obtain

Corollary 4.2 Under the assumptions and hypotheses of Theorem 4.1, we denote by $A_{\varepsilon}$ and $A$, respectively, the linear unbounded selfadjoint operators in $L^{2}(\Omega)$ induced by the bilinear forms $a_{\varepsilon}(\cdot, \cdot)$ and $a_{0}(\cdot, \cdot)$ above.

Then, a point in the resolvent set $\xi \in \rho(A)$ also satisfies $\xi \in \rho\left(A_{\varepsilon}\right)$ for small $\varepsilon$, and

$$
\left\|\left(A_{\varepsilon}-\xi I\right)^{-1}-(A-\xi I)^{-1}\right\|_{\mathcal{L}\left(L^{2}(\Omega), H^{1}(\Omega)\right)} \rightarrow 0, \quad \varepsilon \rightarrow 0 .
$$

Even more, if $\gamma$ denotes a simple closed curve contained in $\rho(A)$, then the corresponding projection

$$
P_{\gamma}\left(A_{\varepsilon}\right)=\frac{1}{2 \pi i} \int_{\gamma}\left(A_{\varepsilon}-\mu I\right)^{-1} d \mu
$$

converges to $P_{\gamma}(A)$ as $\varepsilon \rightarrow 0$ in $\mathcal{L}\left(L^{2}(\Omega), H^{1}(\Omega)\right)$. Moreover, if $\mu_{0}$ is an isolated eigenvalue of $A$ with finite multiplicity $m$, then any curve $\gamma$ enclosing $\mu_{0}$ but not other point of $\sigma(A)$ encloses, for sufficiently small $\varepsilon$, eigenvalues of $A_{\varepsilon}$ with total multiplicity $m$.

Proof. Using Theorem 4.1 we obtain that the resolvents converge in norm at some point $\mu$, i.e.

$$
\left\|\left(A_{\varepsilon}-\mu I\right)^{-1}-(A-\mu I)^{-1}\right\|_{\mathcal{L}\left(L^{2}(\Omega), H^{1}(\Omega)\right)} \rightarrow 0, \quad \varepsilon \rightarrow 0 .
$$

Therefore, from the theory developed in [10] or applying directly Theorem 9.10 in [13] we conclude the spectral convergence of the corollary.

Remark 4.3 i) The convergence of the spectra given by Corollary 4.2 can be restated in the following way: if $\left\{\lambda_{n}^{\varepsilon}\right\}_{n=1}^{\infty}$ is the set of eigenvalues of $A_{\varepsilon}$, for $0 \leq \varepsilon \leq \varepsilon_{0}$, with $\lambda_{1}^{\varepsilon} \leq \lambda_{2}^{\varepsilon} \leq \ldots \lambda_{n}^{\varepsilon}$ and counting multiplicity then, for all $n=1,2, \ldots$,

$$
\lambda_{n}^{\varepsilon} \rightarrow \lambda_{n}^{0} \quad \text { as } \quad \varepsilon \rightarrow 0 .
$$

Moreover, if we denote by $\left\{\varphi_{n}^{\varepsilon}\right\}_{n=1}^{\infty}$ a corresponding set of orthonormal eigenfunctions of $A_{\varepsilon}$, if $\lambda_{n_{1}}^{0}<\lambda_{n}^{0}=\ldots=\lambda_{m}^{0}<\lambda_{m+1}^{0}$ and if we consider the finite dimensional spaces $U_{\varepsilon}=\operatorname{span}\left\{\varphi_{n}^{\varepsilon}, \ldots, \varphi_{m}^{\varepsilon}\right\}$ for $0 \leq \varepsilon \leq \varepsilon_{0}$, then $U_{\varepsilon} \rightarrow U_{0}$ in the sense that the unit balls of $U_{\varepsilon}$ converge in the symmetric Haussdorf distance to the unit ball of $U_{0}$.

ii) From the Corollary above the following can also be obtained. Assume $\left\{\lambda_{n}^{\varepsilon}\right\}_{n=1}^{\infty}$ is the set of eigenvalues of $A_{\varepsilon}$, for $0 \leq \varepsilon \leq \varepsilon_{0}$, with $\lambda_{1}^{\varepsilon} \leq \lambda_{2}^{\varepsilon} \leq \ldots \lambda_{n}^{\varepsilon}$ and counting multiplicity. Also, assume for each $\varepsilon$ and $n$ an associated eigenfunction is chosen such that $\left\{\varphi_{n}^{\varepsilon}(x)\right\}_{n=1}^{\infty}$ is a Hilbert basis of $L^{2}(\Omega)$. 
Then there exists a subsequence, that we still denote $\varepsilon \rightarrow 0$ such that for every $n \in \mathbb{N}$,

$$
\varphi_{n}^{\varepsilon}(x) \rightarrow \varphi_{n}(x) \quad \text { in } \quad H^{1}(\Omega)
$$

and $\left\{\varphi_{n}(x)\right\}_{n=1}^{\infty}$ is a Hilbert basis of $L^{2}(\Omega)$ of eigenfunctions of $\left\{\lambda_{n}^{0}\right\}_{n=1}^{\infty}$.

iii) Note that the spectral convergence above also apply in case the coefficients of the elliptic operators are smooth, as in Section 3.

Now we will show that under natural but more restrictive assumptions than in Theorem 4.1, we can actually conclude the uniform convergence of solutions, that is

$$
u^{\varepsilon} \rightarrow u \quad \text { in } C(\bar{\Omega}) \text {. }
$$

For this we will use the classical De Giorgi-Moser technique; see [11] and we follow closely the approach in [4].

Our first result shows that under suitable conditions, the solutions $u^{\varepsilon}$ are uniformly bounded in $L^{\infty}(\Omega)$. Note that, even if $V^{\varepsilon}=0$ this result does not follow straight from [11] nor [4], since in such cases one needs the right hand side to be uniformly bounded in $L^{p}(\Omega)$ for $p>N / 2$, which is not the case here, since we only have uniform bounds in $L^{1}(\Omega)$.

Theorem 4.4 Under the above assumptions and notations and assuming $r, \rho, t>N-1$ and $z>N / 2$, we have $u^{\varepsilon} \in L^{\infty}(\Omega)$, and

$$
\left\|u^{\varepsilon}\right\|_{L^{\infty}(\Omega)} \leq C_{1}
$$

for some constant $C_{1}>0$ independent of $\varepsilon$ and depending on $s, p, r, \rho, N$, $\|b\|_{L^{s}},\|c\|_{L^{p}},\left\|g_{\varepsilon}\right\|_{L^{z}},\left\|j_{\varepsilon}\right\|_{L^{t}\left(\Gamma_{1}\right)}$.

Proof. We proceed in several steps.

Step 1 We will prove first that $u_{\varepsilon} \in L^{y}(\Omega)$ for all $1 \leq y<\infty$ and that $\left\|u_{\varepsilon}\right\|_{L^{y}(\Omega)} \leq C(y)$ for some constant $C(y)$ independent of $\varepsilon$. and

For this, we will show that for any $\frac{2 N}{N+2} \leq d<\frac{N}{2}$, then $u^{\varepsilon} \in L^{\frac{d N}{N-2 d}}(\Omega)$

$$
\left\|u^{\varepsilon}\right\|_{L^{\frac{d N}{N-2 d}(\Omega)}} \leq C(d)
$$

for some constant $C$ independent of $\varepsilon$. Throughout the proof we will denote $m=\inf _{\Omega} a$.

Note first that for $\frac{2 N}{N+2} \leq d<\frac{N}{2}$, we have that $q=\frac{(N-2) d}{N-2 d} \geq 2$. If we multiply the equation by $\left|u_{\varepsilon}\right|^{q-2} u_{\varepsilon}$ and integrate by parts we obtain

$$
\begin{aligned}
\left.\left.\frac{4(q-1)}{q^{2}} \int_{\Omega} a|\nabla| u_{\varepsilon}\right|^{\frac{q}{2}}\right|^{2} & +\int_{\Omega}(c+\lambda)\left(\left|u_{\varepsilon}\right|^{\frac{q}{2}}\right)^{2}+\int_{\Gamma} b\left(\left|u_{\varepsilon}\right|^{\frac{q}{2}}\right)^{2}+\frac{1}{\varepsilon} \int_{\omega_{\varepsilon}} V_{\varepsilon}\left(\left|u_{\varepsilon}\right|^{\frac{q}{2}}\right)^{2} \\
& =\frac{1}{\varepsilon} \int_{\omega_{\varepsilon}} f_{\varepsilon}\left|u_{\varepsilon}\right|^{q-2} u_{\varepsilon}+\int_{\Gamma} j_{\varepsilon}\left|u_{\varepsilon}\right|^{q-2} u_{\varepsilon}+\int_{\Omega} g_{\varepsilon}\left|u_{\varepsilon}\right|^{q-2} u_{\varepsilon} .
\end{aligned}
$$


Denoting by $v_{\varepsilon}=\left|u_{\varepsilon}\right|^{\frac{q}{2}}$, we have

$$
\begin{aligned}
\left.\left|\int_{\Omega} g_{\varepsilon}\right| u_{\varepsilon}\right|^{q-2} u_{\varepsilon} \mid & \leq\left[\int_{\Omega}\left|g_{\varepsilon}\right|^{d}\right]^{\frac{1}{d}}\left[\int_{\Omega}\left|u_{\varepsilon}\right|^{(q-1) d^{\prime}}\right]^{\frac{1}{d^{\prime}}}= \\
& =\left\|g_{\varepsilon}\right\|_{L^{d}}\left[\int_{\Omega}\left|v^{\varepsilon}\right|^{\frac{2 d^{\prime}}{q^{\prime}}}\right]^{\frac{1}{d^{\prime}}}=\left\|g_{\varepsilon}\right\|_{L^{d}(\Omega)}\left\|v^{\varepsilon}\right\|_{L^{\frac{2 d^{\prime}}{q^{\prime}}}(\Omega)}^{\frac{2}{q^{\prime}}} .
\end{aligned}
$$

Now, since $\frac{2 d^{\prime}}{q^{\prime}}=\frac{2 N}{N-2}$ we have

$$
\left.\left|\int_{\Omega} g_{\varepsilon}\right| u_{\varepsilon}\right|^{q-2} u_{\varepsilon} \mid \leq C\left\|v_{\varepsilon}\right\|_{H^{1}(\Omega)}^{\frac{2}{q^{\prime}}}
$$

If we denote by $n=\frac{(N-1) d}{N-d}<N-1$, we have

$\left.\left|\frac{1}{\varepsilon} \int_{\omega_{\varepsilon}} f_{\varepsilon}\right| u_{\varepsilon}\right|^{q-2} u_{\varepsilon} \mid \leq\left[\frac{1}{\varepsilon} \int_{\omega_{\varepsilon}}\left|f_{\varepsilon}\right|^{n}\right]^{\frac{1}{n}}\left[\frac{1}{\varepsilon} \int_{\omega_{\varepsilon}}\left|u_{\varepsilon}\right|^{(q-1) n^{\prime}}\right]^{\frac{1}{n^{\prime}}} \leq C_{0}\left[\frac{1}{\varepsilon} \int_{\omega_{\varepsilon}}\left|v_{\varepsilon}\right|^{\frac{2 n^{\prime}}{q^{\prime}}}\right]^{\frac{1}{n^{\prime}}}$.

Now, since $\frac{2 n^{\prime}}{q^{\prime}}=\frac{2(N-1)}{N-2}$, we have, using Lemma 2.1, that

$$
\left.\left|\frac{1}{\varepsilon} \int_{\omega_{\varepsilon}} f_{\varepsilon}\right| u_{\varepsilon}\right|^{q-2} u_{\varepsilon} \mid \leq C_{0}\left[\frac{1}{\varepsilon} \int_{\omega_{\varepsilon}}\left|v_{\varepsilon}\right|^{\frac{2(N-1)}{N-2}}\right]^{\frac{N-2}{2(N-1)} \cdot \frac{2}{q^{\prime}}} \leq C\left\|v_{\varepsilon}\right\|_{H^{1}(\Omega)}^{\frac{2}{q^{\prime}}} .
$$

In a similar way, we have

$$
\left.\left|\int_{\Gamma} j_{\varepsilon}\right| u_{\varepsilon}\right|^{q-2} u_{\varepsilon} \mid \leq\left[\int_{\Gamma}\left|j_{\varepsilon}\right|^{n}\right]^{\frac{1}{n}}\left[\int_{\Gamma}\left|u_{\varepsilon}\right|^{(q-1) n^{\prime}}\right]^{\frac{1}{n^{\prime}}}=\left\|j_{\varepsilon}\right\|_{L^{n}(\Gamma)}\left[\frac{1}{\varepsilon} \int_{\Gamma}\left|v_{\varepsilon}\right|^{\frac{2 n^{\prime}}{q^{\prime}}}\right]^{\frac{1}{n^{\prime}}}
$$

and using again that $\frac{2 n^{\prime}}{q^{\prime}}=\frac{2(N-1)}{N-2}$, we get

$$
\left.\left|\int_{\Gamma} j_{\varepsilon}\right| u_{\varepsilon}\right|^{q-2} u_{\varepsilon} \mid \leq C\left\|v_{\varepsilon}\right\|_{H^{1}(\Omega)}^{\frac{2}{q^{\prime}}}
$$

Putting all these estimates together, we have

$$
\frac{4(q-1)}{q^{2}} \int_{\Omega} a\left|\nabla v_{\varepsilon}\right|^{2}+\int_{\Omega}(c+\lambda) v_{\varepsilon}^{2}+\int_{\Gamma} b v_{\varepsilon}^{2}+\frac{1}{\varepsilon} \int_{\omega_{\varepsilon}} V_{\varepsilon}\left|v_{\varepsilon}\right|^{2} \leq C\left\|v_{\varepsilon}\right\|_{H^{1}(\Omega)}^{\frac{2}{q^{\top}}} .
$$

We can add to both sides of the previous inequality a term of the type $\mu \int_{\Omega} v_{\varepsilon}^{2}$, with $\mu=\mu(q)$, so that the left hand side is uniformly coercive, obtaining,

$$
C(q)\left\|v_{\varepsilon}\right\|_{H^{1}(\Omega)}^{2} \leq C\left\|v_{\varepsilon}\right\|_{H^{1}(\Omega)}^{\frac{2}{q^{\prime}}}+C\left\|v_{\varepsilon}\right\|_{L^{2}(\Omega)}^{2} .
$$


Hence,

$$
\begin{aligned}
C(q)\left\|v_{\varepsilon}\right\|_{H^{1}(\Omega)}^{2} & \leq C\left\|v_{\varepsilon}\right\|_{H^{1}(\Omega)}^{\frac{2}{q^{\prime}}}+C\left\|v_{\varepsilon}\right\|_{L^{2}(\Omega)}^{2} \\
& \leq C\left\|v_{\varepsilon}\right\|_{H^{1}(\Omega)}^{\frac{2}{q^{\prime}}}+C\left\|v_{\varepsilon}\right\|_{H^{1}(\Omega)}^{\frac{2}{q^{\prime}}}\left\|v_{\varepsilon}\right\|_{L^{q}(\Omega)}^{\frac{2}{q}} .
\end{aligned}
$$

From where we get

$$
\left\|v_{\varepsilon}\right\|_{L^{\frac{2 N}{N-2}}(\Omega)}^{\frac{2}{q}} \leq C(q)\left(1+\left\|u_{\varepsilon}\right\|_{L^{q}(\Omega)}\right)
$$

and since $v_{\varepsilon}=\left|u_{\varepsilon}\right|^{\frac{q}{2}}$ and $q=\frac{(N-2) d}{N-2 d}$, we get

$$
\left\|u_{\varepsilon}\right\|_{L^{q+\frac{2 d}{N-2 d}(\Omega)}} \leq C(q)\left(1+\left\|u_{\varepsilon}\right\|_{L^{q}(\Omega)}\right) .
$$

and since $\frac{2 d}{N-2 d} \geq \frac{2}{N-2}$ we get

$$
\left\|u_{\varepsilon}\right\|_{L^{q+\frac{2}{N-2}(\Omega)}} \leq C(q)\left(1+\left\|u_{\varepsilon}\right\|_{L^{q}(\Omega)}\right) .
$$

Applying a bootstrap argument, starting at $q=2$ we obtain that $u_{\varepsilon} \in$ $L^{q}(\Omega)$ for all $q<\infty$ and that

$$
\left\|u_{\varepsilon}\right\|_{L^{q}(\Omega)} \leq C
$$

where $C$ may depend on $q$ but it is independent of $\varepsilon$.

Step 2 We now prove that we have $u^{\varepsilon} \in L^{\infty}(\Omega)$ and

$$
\left\|u^{\varepsilon}\right\|_{L^{\infty}(\Omega)} \leq C
$$

for some constant $C$ independent of $\varepsilon$.

Let $k>0, \phi_{\varepsilon}=\left(u^{\varepsilon}-k\right)^{+} \leq\left|u_{\varepsilon}\right|$ and $A_{k}=\left\{x \in \Omega: u^{\varepsilon}(x)>k\right\}$, where we drop the dependence of $\varepsilon$ in these sets to simplify the notations. Notice first that, from Step 1 , for any $q \geq 2$ the following inequality holds

$$
\left\|\phi_{\varepsilon}\right\|_{L^{q}(\Omega)} \leq\left\|u_{\varepsilon}\right\|_{L^{q}(\Omega)} \leq C
$$

where $C$ is independent of $\varepsilon$ and $k$, but may depend on $q$. Now note that using interpolation we have

$$
\begin{aligned}
\left\|\phi_{\varepsilon}\right\|_{L^{2}(\Omega)}^{2} & \leq\left\|\phi_{\varepsilon}\right\|_{L^{q}(\Omega)}\left|A_{k}\right|^{\frac{1}{2}-\frac{1}{q}}\left\|\phi_{\varepsilon}\right\|_{L^{\frac{2 N}{N-2}}(\Omega)}\left|A_{k}\right|^{\frac{1}{N}} \\
& \leq C\left\|\phi_{\varepsilon}\right\|_{L^{q}(\Omega)}\left\|\phi_{\varepsilon}\right\|_{H^{1}(\Omega)}\left|A_{k}\right|^{\frac{1}{2}+\frac{1}{N}-\frac{1}{q}}
\end{aligned}
$$


From here, taking $q=2 N$ and using (4.23), we obtain

$$
\left\|\phi_{\varepsilon}\right\|_{L^{2}(\Omega)}^{2} \leq C\left\|\phi_{\varepsilon}\right\|_{H^{1}(\Omega)}\left|A_{k}\right|^{\frac{1}{2}+\frac{1}{2 N}} .
$$

Using $\phi_{\varepsilon}$ as a test function in the equation we have, for $\lambda>0$,

$$
\begin{aligned}
& \int_{\Omega} a\left|\nabla \phi_{\varepsilon}\right|^{2}+\int_{\Omega}(c+\lambda) \phi_{\varepsilon}^{2}+\int_{\Gamma} b \phi_{\varepsilon}^{2}+\frac{1}{\varepsilon} \int_{\omega_{\varepsilon}} V_{\varepsilon}\left|\phi_{\varepsilon}\right|^{2} \\
& 25)=\frac{1}{\varepsilon} \int_{\omega_{\varepsilon}} f_{\varepsilon} \phi_{\varepsilon}+\int_{\Gamma}\left(j_{\varepsilon}-k b\right) \phi_{\varepsilon}+\int_{\Omega}\left[g_{\varepsilon}-k(c+\lambda)\right] \phi_{\varepsilon}-\frac{1}{\varepsilon} \int_{\omega_{\varepsilon}} k V_{\varepsilon} \phi_{\varepsilon} .
\end{aligned}
$$

Next we estimate each term on the right hand side of the above expression, for this, we use extensively Holder's inequality, Sobolev embeddings and trace theorems.

Now, denoting $g_{\varepsilon}^{*}=g_{\varepsilon}-k(c+\lambda)$ and taking $N / 2<p_{0} \leq p, z$, we have

$$
\begin{aligned}
\left|\int_{\Omega} g_{\varepsilon}^{*} \phi_{\varepsilon}\right| & \leq\left\|g_{\varepsilon}^{*}\right\|_{L^{p_{0}(\Omega)}}\left\|\phi_{\varepsilon}\right\|_{L^{p_{0}^{\prime}(\Omega)}} \leq C(1+k)\left\|\phi_{\varepsilon}\right\|_{L^{\frac{2 N}{N-2}}\left(A_{k}\right)}\left|A_{k}\right|^{\frac{1}{2}-\frac{1}{p_{0}}+\frac{1}{N}} \\
& \leq C(1+k)\left\|\phi_{\varepsilon}\right\|_{H^{1}(\Omega)}\left|A_{k}\right|^{\frac{1}{2}-\frac{1}{p_{0}}+\frac{1}{N}} .
\end{aligned}
$$

On the other hand, using Lemma 2.1

$$
\begin{aligned}
\left|\frac{1}{\varepsilon} \int_{\omega_{\varepsilon}} f_{\varepsilon} \phi_{\varepsilon}\right| & \leq\left[\frac{1}{\varepsilon} \int_{\omega_{\varepsilon}}\left|f_{\varepsilon}\right|^{r}\right]^{\frac{1}{r}}\left[\frac{1}{\varepsilon} \int_{\omega_{\varepsilon}}\left|\phi_{\varepsilon}\right|^{r^{\prime}}\right]^{\frac{1}{r^{\prime}}} \\
& \leq C_{0}\left\|\phi_{\varepsilon}\right\|_{H^{1, \frac{N r^{\prime}}{N+r^{\prime}-1}(\Omega)}}=C_{0}\left\|\phi_{\varepsilon}\right\|_{H^{1, \frac{N r}{N r-N+1}(\Omega)}} .
\end{aligned}
$$

Hence, using $\frac{N r}{N r-N+1}<2$ we get

$$
\left|\frac{1}{\varepsilon} \int_{\omega_{\varepsilon}} f_{\varepsilon} \phi_{\varepsilon}\right| \leq C\left\|\phi_{\varepsilon}\right\|_{H^{1}(\Omega)}\left|A_{k}\right|^{\frac{1}{2}-\frac{1}{r}+\frac{1}{N r}}
$$

and in a similar way we get

$$
\left|\frac{1}{\varepsilon} \int_{\omega_{\varepsilon}} V_{\varepsilon} k \phi_{\varepsilon}\right| \leq C k\left\|\phi_{\varepsilon}\right\|_{H^{1}(\Omega)}\left|A_{k}\right|^{\frac{1}{2}-\frac{1}{r}+\frac{1}{N r}} .
$$

For the terms on the boundary, if we note that $j_{\varepsilon}^{*}=j_{\varepsilon}-k b$ and we choose a $N-1<s_{0}<s, t$, we have

$$
\begin{aligned}
\left|\int_{\Gamma} j_{\varepsilon}^{*} \phi_{\varepsilon}\right| & \leq\left[\int_{\Gamma}\left|j_{\varepsilon}^{*}\right|^{s_{0}}\right]^{\frac{1}{s_{0}}}\left[\int_{\Gamma}\left|\phi_{\varepsilon}\right|^{s_{0}^{\prime}}\right]^{\frac{1}{s_{0}^{\prime}}} \\
& \leq C(1+k)\left\|\phi_{\varepsilon}\right\|_{L^{s_{0}^{\prime}(\Gamma)}} \leq C(1+k)\left\|\phi_{\varepsilon}\right\|_{H^{1,} \frac{N s_{0}}{N s_{0}-N+1}(\Omega)}
\end{aligned}
$$


and using again $\frac{N s_{0}}{N s_{0}-N+1}<2$ and proceeding as for (4.26), we get

$$
\left|\int_{\Gamma} j_{\varepsilon}^{*} \phi_{\varepsilon}\right| \leq C(1+k)\|\phi\|_{H^{1}(\Omega)}\left|A_{k}\right|^{\frac{1}{2}-\frac{N-1}{s_{0} N}}
$$

Now, using the estimates above and choosing $\delta$ enough small, we obtain

$$
\begin{aligned}
& \frac{m}{2}\left\|\phi_{\varepsilon}\right\|_{H^{1}(\Omega)}^{2} \leq \int_{\Omega} a\left|\nabla \phi_{\varepsilon}\right|^{2}+\lambda \int_{\Omega} \phi_{\varepsilon}^{2} \\
& \leq C(1+k)\left\|\phi_{\varepsilon}\right\|_{H^{1}(\Omega)} \\
& \quad \times\left(\left|A_{k}\right|^{\frac{1}{2}-\frac{1}{r}+\frac{1}{N r}}+\left|A_{k}\right|^{\frac{1}{2}-\frac{1}{s_{0}}+\frac{1}{N s_{0}}}+\left|A_{k}\right|^{\frac{1}{2}+\frac{1}{N}-\frac{1}{p_{0}}}+\left|A_{k}\right|^{\frac{1}{2}+\frac{1}{N}}\right)
\end{aligned}
$$

while we also have

$$
\left\|\phi_{\varepsilon}\right\|_{L^{1}\left(A_{k}\right)} \leq\left\|\phi_{\varepsilon}\right\|_{L^{\frac{2 N}{N-2}}\left(A_{k}\right)}\left|A_{k}\right|^{\frac{N+2}{2 N}} \leq C\left\|\phi_{\varepsilon}\right\|_{H^{1}(\Omega)}\left|A_{k}\right|^{\frac{N+2}{2 N}},
$$

where $C=C(\Omega, N)$. With these, we get that

$$
\left\|\phi_{\varepsilon}\right\|_{L^{1}(\Omega)} \leq \gamma(1+k)\left|A_{k}\right|^{1+\alpha}
$$

where

$$
\alpha=\min \left\{\frac{r-(N-1)}{N r}, \frac{s_{0}-(N-1)}{N s_{0}}, \frac{2 p_{0}-N}{N p_{0}}, \frac{2}{N}\right\}>0 .
$$

In particular, for $k \geq 1$ we have

$$
\left\|\phi_{\varepsilon}\right\|_{L^{1}(\Omega)} \leq 2 \gamma k\left|A_{k}\right|^{1+\alpha} .
$$

Now, using the Lemma 5.1 in [11] we have that

$$
\max \left\{u^{\varepsilon}(x) ; x \in \Omega\right\} \leq C .
$$

With a similar argument for $w_{\varepsilon}=-u^{\varepsilon}$, we obtain the desired result.

Remark 4.5 Let us observe that to obtain the results of Theorem 4.4, that is, the uniform boundedness of $u^{\varepsilon}$ in $L^{\infty}(\Omega)$ we do not need the convergence hypotheses of (4.3) and (4.4), but it will be sufficient to guarantee the boundedness hypothesis given by (4.2) and to assume that the family $g_{\varepsilon}$ and $j_{\varepsilon}$ are bounded in $L^{z}(\Omega)$ and $L^{t}(\Gamma)$ with the appropriate $z$ and $t$.

Now we can show the uniform convergence.

Theorem 4.6 Under the conditions above, we have

$$
u^{\varepsilon} \rightarrow u \quad \text { in } L^{\infty}(\Omega) .
$$


Proof. Note that $v_{\varepsilon}=u^{\varepsilon}-u$ satisfies

$$
\left\{\begin{aligned}
-\operatorname{div}\left(a(x) \nabla v_{\varepsilon}\right)+c(x) v_{\varepsilon}+\lambda v_{\varepsilon}+\frac{1}{\varepsilon} \mathcal{X}_{\omega_{\varepsilon}} V_{\varepsilon}\left(v_{\varepsilon}+u\right) & =\frac{1}{\varepsilon} \mathcal{X}_{\omega_{\varepsilon}} f_{\varepsilon}+g_{\varepsilon}-g_{0} & & \text { in } \Omega \\
a(x) \frac{\partial v_{\varepsilon}}{\partial n}+b(x) v_{\varepsilon} & =-f_{0}-V_{0} u & & \text { on } \Gamma \\
v_{\varepsilon} & =0, & & \text { on } \Gamma_{D} .
\end{aligned}\right.
$$

As in Theorem 4.4 if $A_{k}=\left\{x \in \Omega: v_{\varepsilon}>k\right\}$, we obtain

$$
\left\|\left(v_{\varepsilon}-k\right)^{+}\right\|_{L^{1}(\Omega)} \leq \gamma(1+k)\left|A_{k}\right|^{1+\alpha}, \text { for every } k>0
$$

From (4.31) we get that there exists a constant $K_{0}$ independent of $\varepsilon$ such that $\left\|v_{\varepsilon}^{+}\right\|_{L^{\infty}(\Omega)} \leq K_{0}$ uniformly in $\varepsilon$. Hence, if $0<k \leq K_{0}$ we have

$$
\left\|\left(v_{\varepsilon}-k\right)^{+}\right\|_{L^{1}(\Omega)} \leq \gamma\left(1+K_{0}\right)\left|A_{k}\right|^{1+\alpha}, \text { for every } 0<k<K_{0}
$$

and if $k>K_{0}$ we have $\left(v_{\varepsilon}-k\right)^{+} \equiv 0$. Hence we can assure that we always have for $\tilde{\gamma}=\gamma\left(1+K_{0}\right)$ that

$$
\left\|\left(v_{\varepsilon}-k\right)^{+}\right\|_{L^{1}(\Omega)} \leq \tilde{\gamma}\left|A_{k}\right|^{1+\alpha}, \text { for every } k>0 .
$$

Since for every $k>k_{0}>0$ we have $\left|A_{k}\right| \leq\left|A_{k_{0}}\right|$, then

$$
\left\|\left(v_{\varepsilon}-k\right)^{+}\right\|_{L^{1}(\Omega)} \leq \tilde{\gamma}\left|A_{k_{0}}\right|^{\frac{\alpha}{2}}\left|A_{k}\right|^{1+\frac{\alpha}{2}} \text {, for every } k>k_{0}
$$

and using Lemma 5.1 from [11] we get

$$
\left\|v_{\varepsilon}\right\|_{L^{\infty}(\Omega)} \leq k_{0}+C\left|A_{k_{0}}\right|^{\frac{\alpha}{\alpha+2}} .
$$

where $C$ is independent of $k_{0}, k$ and $\varepsilon$. Now, observe that the convergence in Theorem 4.1 implies that $\left|A_{k_{0}}\right| \rightarrow 0$ as $\varepsilon \rightarrow 0$. Since $k_{0}$ is arbitrarily small we get the result.

As in the case of smooth coefficients, analyzed in the previous section, we can also obtain the convergence in the case where we have a nonhomogeneous Dirichlet boundary condition in $\Gamma_{D}$. We have

Corollary 4.7 Assume we are in the conditions of Theorem 4.6. Let $h$ be a function defined in $\Gamma_{D}$ such that $h \in H^{1 / 2}\left(\Gamma_{D}\right) \cap L^{\infty}\left(\Gamma_{D}\right)$. Then if in problems (1.4) and (1.5) we substitute the condition $u=0$ in $\Gamma_{D}$ by $u=h$ in $\Gamma_{D}$, then the convergence result of Theorem 4.6 also holds.

Proof. The proof follows the same ideas as the one from Corollary 3.3. 


\section{Final remarks}

We present in this section some important remarks related to the results obtained in the preceding sections.

Remark 5.1 Note that the convergence results in Sections 3 and 4 for (1.4) and (1.5) can be seen as a tool for transfering information from the interior to the boundary. In particular, the results above, allow to approximate the solution of the particular case of (1.5)

$$
\begin{cases}-\operatorname{div}(a(x) \nabla u)+c(x) u+\lambda u=g & \text { in } \Omega \\ a(x) \frac{\partial u}{\partial n}+V_{0}(x) u=f_{0} & \text { on } \Gamma=\partial \Omega\end{cases}
$$

by the solutions of the concentrated problem

$$
\left\{\begin{array}{lr}
-\operatorname{div}\left(a(x) \nabla u^{\varepsilon}\right)+c(x) u^{\varepsilon}+\lambda u^{\varepsilon}+\frac{1}{\varepsilon} \mathcal{X}_{\omega_{\varepsilon}} V_{\varepsilon}(x) u^{\varepsilon}=g+\frac{1}{\varepsilon} \mathcal{X}_{\omega_{\varepsilon}} f_{\varepsilon} \text { in } \Omega \\
\frac{\partial u}{\partial n}=0 & \text { on } \Gamma=\partial \Omega
\end{array}\right.
$$

As a matter of fact, we may define $V_{\varepsilon}$ and $f_{\varepsilon}$ extending the functions $V_{0}$ and $f_{0}$ to $\omega_{\varepsilon}$ in the direction of the normal. That is, if $z \in \omega_{\varepsilon}$ then $z$ can be written in a unique way as $z=x-\sigma \vec{n}(x)$, for some $\sigma \in(0, \varepsilon)$. Therefore, we define $V_{\varepsilon}(z)=V_{0}(x)$ and $f_{\varepsilon}(z)=f_{0}(x)$. With this definition we may easily prove that if $V_{0} \in L^{\rho}(\Gamma), f_{0} \in L^{r}(\Gamma)$, then $V_{\varepsilon} \in L^{\rho}\left(\omega_{\varepsilon}\right), f_{\varepsilon} \in L^{r}\left(\omega_{\varepsilon}\right)$ and that both (1.2) and (1.3) hold. If we also assume $r, \rho>N-1, g \in L^{z}(\Omega)$ with $z>N / 2$ and $\lambda$ is large enough, then we may apply the results of Section 3 if we are in the case of smooth coefficients $a, b, c$ or we may apply the results of Section 4 if $a, b$ and $c$ are not smooth and in both cases we obtain the convergence of the solution of (5.2) to the solution of (5.1), see Corollary 3.2 and Theorem 4.6.

In other words, nonhomogeneous Robin problems can be approximated by homogeneous Neumann problems efficiently. Note that this leads to some applications for numerical approximations, since Neumann conditions are easier to implement; see [8].

Remark 5.2 Observe that in case the domain is not smooth, it may be difficult to give a meaning to the boundary condition in (5.1), although (5.2) has a natural and simple variational formulation not involving surface integrals or traces. Hence the limit functions of (5.2) can be taken as proper way of defining solutions of (5.1). 
Remark 5.3 It is not difficult to see that all the results from Sections 3 and 4 can be carried out with minor changes to the case in which $\omega_{\varepsilon}$ collapses to a regular hyper-surface $\Gamma \subset \bar{\Omega}$, not necessarily the boundary of the domain. In such a case, for the problem

$$
\left\{\begin{aligned}
-\operatorname{div}\left(a(x) \nabla u^{\varepsilon}\right)+c(x) u^{\varepsilon}+\lambda u^{\varepsilon}+\frac{1}{\varepsilon} \mathcal{X}_{\omega_{\varepsilon}} V_{\varepsilon} u^{\varepsilon} & =\frac{1}{\varepsilon} \mathcal{X}_{\omega_{\varepsilon}} f_{\varepsilon}+g & & \text { in } \Omega \\
a(x) \frac{\partial u^{\varepsilon}}{\partial n}+b(x) u^{\varepsilon} & =j & & \text { on } \partial \Omega_{R} \\
u^{\varepsilon} & =0 & & \text { on } \partial \Omega_{D}
\end{aligned}\right.
$$

the limit problem reads

$$
\left\{\begin{aligned}
-\operatorname{div}(a(x) \nabla u)+c(x) u+\lambda u+V_{0} \delta_{\Gamma} u & =f_{0} \delta_{\Gamma}+g & & \text { in } \Omega \\
a(x) \frac{\partial u}{\partial n}+b(x) u & =j & & \text { on } \partial \Omega_{R} \\
u & =0 & & \text { on } \partial \Omega_{D}
\end{aligned}\right.
$$

where we denote by $f_{0} \delta_{\Gamma}$ and $V_{0} \delta_{\Gamma} u$ the functionals

$$
<f_{0} \delta_{\Gamma}, \varphi>=\int_{\Gamma} f_{0} \varphi \quad \text { and } \quad<V_{0} u, \varphi>=\int_{\Gamma} V_{0} u \varphi .
$$

We also denote by $\partial \Omega_{R}$ and $\partial \Omega_{D}$ two disjoint sets of $\partial \Omega$ where Robin and Dirichlet type boundary condition are imposed, respectively.

By this we mean that if $V_{\varepsilon}, f_{\varepsilon}, V_{0}$ and $f_{0}$ satisfy (1.2) and (1.3), then if $\rho>N-1, r>N-1, g \in L^{z}(\Omega)$ with $z>N / 2$ and $\lambda$ is large enough, then Corollary 3.2 holds for the case of smooth coefficients $a, b$ and $c$ and Theorem 4.6 holds for the nonsmooth coefficients case, where $u_{\varepsilon}$ is the solution of (5.3) and $u$ is the solution of (5.4).

Observe also that by taking test functions with support near points on $\Gamma$ it is easy to see that the limit problem (5.4) is in fact a transmission problem across $\Gamma$, where the jump condition reads

$$
[u]_{\Gamma}=0, \quad\left[a(x) \frac{\partial u}{\partial n}+V_{0}(x) u\right]_{\Gamma}=f_{0} .
$$

Hence, in particular, the solution of the transmission problem

$$
\begin{cases}-\operatorname{div}(a(x) \nabla u)+c(x) u+\lambda u=g & \text { in } \Omega \\ {[u]_{\Gamma}=0, \quad\left[a(x) \frac{\partial u}{\partial n}+V_{0}(x) u\right]_{\Gamma}=f_{0}} & \text { on } \Gamma \\ u=0 & \text { on } \partial \Omega\end{cases}
$$

can be approximated by the solutions of the concentrated problems

$$
\begin{cases}-\operatorname{div}\left(a(x) \nabla u^{\varepsilon}\right)+c(x) u^{\varepsilon}+\lambda u^{\varepsilon}+\frac{1}{\varepsilon} \mathcal{X}_{\omega_{\varepsilon}} V_{\varepsilon} u^{\varepsilon}=g+\frac{1}{\varepsilon} \mathcal{X}_{\omega_{\varepsilon}} f_{\varepsilon} & \text { in } \Omega \\ u^{\varepsilon}=0 & \text { on } \partial \Omega\end{cases}
$$


This approach may lead to significant applications to numerical schemes for such problems.

In this direction observe that (1.4) and (5.3) can be understood as approximate regularized problems for (1.5) and (5.4) respectively.

\section{References}

[1] Adams, R.: Sobolev Spaces. Pure and Applied Mathematics 65. Academic Press, New York-London, 1975.

[2] Amann, H.: Nonhomogeneous linear and quasilinear elliptic and parabolic boundary value problems. In Function spaces, differential operators and nonlinear analysis (Friedrichroda, 1992), 9-126. Teubner-Texte Math. 133. Teubner, Stuttgart, 1993.

[3] Amann, H.: Linear and quasilinear parabolic problems. Vol. I. Abstract linear theory. Monographs in Mathematics 89. Birkhäuser Boston, Boston, MA, 1995.

[4] Arrieta, J. M., Carvalho, A. N. and Rodríguez-Bernal, A.: Attractors of parabolic problems with nonlinear boundary conditions. Uniform bounds. Comm. Partial Differential Equations 25 (2000), vol. 1-2, 1-37.

[5] Arrieta, J. M., Jiménez-Casas, A. And Rodríguez-Bernal, A.: Flux condition induced by concentraded reactions. In Equadiff 2003, 293-295. World Sci. Publ., Hackensack, NJ, 2005.

[6] Arrieta, J. M., Jiménez-Casas, A. And Rodríguez-Bernal, A.: Robin type conditions arising from concentrated potentials. In Proceedings of Equadiff 11, 157-164. Editors: M. Fila, A. Handlovicova, K. Mikula, M. Medved, P. Quittner and D. Sevcovic. 2007.

[7] Arrieta, J. M., Rodríguez-Bernal, A. And Rossi, J.: The best Sobolev trace constant as limit of the usual Sobolev constant for small strips near the boundary. Proc. Roy. Soc. Edinburgh Sect. A, to appear.

[8] Cónsul, N. And Jorba, A.: On the existence of patterns for a diffusion equation on a convex domain with nonlinear boundary reaction. Internat. J. Bifur. Chaos Appl. Sci. Engrg. 15 (2005), no. 10, 3321-3328.

[9] Gilbarg, D. And Trudinger, N. S.: Elliptic partial differential equations of second order. Grundlehren der Mathematischen Wissenschaften 224. Springer-Verlag, Berlin-New York, 1977.

[10] Kato, T.: Perturbation theory for linear operators. Grundlehren der Mathematischen Wissenschaften 132. Springer-Verlag, Berlin-New York, 1976.

[11] Ladyzhenskaya, O. and Uraltseva, N.: Linear and quasilinear elliptic equations. Academic Press, New York-London, 1968.

[12] Protter, M. And Weinberger, H.: Maximum principles in differential equations. Prentice-Hall, Englewood Cliffs, N.J., 1967. 
[13] Sanchez-Hubert, J. and SÁnchez-Palencia, E.: Vibration and coupling of continuous systems. Asymptotic methods. Springer-Verlag, 1989.

[14] TRIBEL, H.: Interpolation theory, function spaces, differential operators. North Holland, 1978.

Recibido: 29 de mayo de 2006

Revisado: 3 de octubre de 2006

José M. Arrieta

Departamento de Matemática Aplicada Universidad Complutense de Madrid 28040 Madrid, Spain arrieta@mat.ucm.es Ángela Jiménez-Casas Departamento de Matemática Aplicada y Computación Universidad Pontificia Comillas de Madrid

C/ Mártires de Alcalá 11 28015 Madrid, Spain ajimenez@rec.upcomillas.es

Aníbal Rodríguez-Bernal Departamento de Matemática Aplicada Universidad Complutense de Madrid 28040 Madrid, Spain arober@mat.ucm.es

Partially supported by projects BFM2003-03810 and MTM2006-08262, MEC, Spain. Also supported by CCG07-UCM/ESP-2393, UCM-Comunidad de Madrid, Spain. 